\title{
Spectropolarimetry of low redshift Quasars: origin of the polarization and implications for black hole mass estimates.
}

\author{
Alessandro Capetti ${ }^{1 \star}$, Ari Laor $^{2}$, Ranieri D. Baldi $^{3,4}$, Andrew Robinson $^{5}$, Alessandro Marconi ${ }^{6}$ \\ ${ }^{1}$ INAF-Osservatorio Astrofisico di Torino, Strada Osservatorio 20, I-10025, Pino Torinese, Italy \\ ${ }^{2}$ Physics Department, The Technion, 32000, Haifa, Israel \\ ${ }^{3}$ INAF- Istituto di Radioastronomia, Via Gobetti 101, I-40129 Bologna, Italy \\ ${ }^{4}$ Department of Physics and Astronomy, University of Southampton, Highfield, SO17 1BJ, UK \\ ${ }^{5}$ School of Physics and Astronomy, Rochester Institute of Technology, 84 Lomb Memorial Drive, Rochester, NY 14623-5603, USA \\ ${ }^{6}$ Dipartimento di Fisica e Astronomia, Università di Firenze, via G. Sansone 1, 50019 Sesto Fiorentino, Italy
}

12 February 2021

\begin{abstract}
We present the results of high signal-to-noise ratio VLT spectropolarimetry of a representative sample of 25 bright type $1 \mathrm{AGN}$ at $z<0.37$, of which nine are radio-loud. The sample covers uniformly the $5100 \AA$ optical luminosity at $L_{5100} \sim 10^{44}-10^{46} \mathrm{erg} \mathrm{s}^{-1}$, and $\mathrm{H} \alpha$ width at FWHM $\sim 1000-10,000 \mathrm{~km} \mathrm{~s}^{-1}$. We derive the continuum and the $\mathrm{H} \alpha$ polarization amplitude, polarization angle, and angle swing across the line, together with the radio properties. We find the following: 1 . The broad line region (BLR) and continuum polarization are both produced by a single scattering medium. 2 . The scattering medium is equatorial, and at right angle to the system axis. 3. The scattering medium is located at or just outside the BLR. The continuum polarization and the $\mathrm{H} \alpha$ polarization angle swing, can both serve as an inclination indicator. The observed line width is found to be affected by inclination, which can lead to an underestimate of the black hole mass by a factor of $\sim 5$ for a close-to face-on view. The line width measured in the polarized flux overcomes the inclination bias, and provides a close-to equatorial view of the BLR in all AGN, which allows to reduce the inclination bias in the BLR based black hole mass estimates.
\end{abstract}

Key words: galaxies: active-galaxies

\section{INTRODUCTION}

Most AGN are powered by accretion through a disk, and often display a jet. Thus, their emission properties likely have an axial symmetry and can be inclination dependent. If the emission from such a system is polarized, the polarization is inevitably inclination dependent, and should approach zero when the inclination approaches zero (i.e., a face-on view). Thus, polarization may provide a useful inclination indicator in AGN, a point which we explore in this study.

Indeed, spectropolarimetry of type 2 AGN revealed that they harbor an obscured type 1 AGN, which led to the AGN inclination unification scheme (Miller \& Antonucci 1983; Antonucci 1983; Antonucci \& Miller 1985; Antonucci 1993), where a planar toruslike structure obscures the direct light from the Broad Line Region (BLR) and the continuum source at a closer-to edge-on view. A scattering medium, which resides in the polar direction above the obscuring medium, as indicated by polarization angle perpendicular to the jet axis (Antonucci 1983), allows to detect the hidden BLR and continuum source through the scattered polarized light.

* E-mail:alessandro.capetti@inaf.it
Spectropolarimetry of the continuum and broad lines in a variety of type 1 Seyfert galaxies reveals a more elaborate picture, with a wide range of polarization levels and polarization angles (Smith et al. 2005). The polarization in some type 1 AGN is consistent with a polar scattering medium, as observed in type 2 AGN. But, in the majority of type 1 AGN spectropolarimetry suggests an equatorial scattering medium located just outside the BLR, as indicated by the polarization angle and its rotation across the broad line profiles. In addition, some objects show an intermediate scattering medium, or show no evidence for a scattering medium (Goodrich \& Miller 1988, 1989, 1994; Smith et al. 2002, 2004, 2005; Afanasiev et al. 2019).

Spectropolarimetry allows us to constrain the properties and kinematics of the scattering medium, and furthermore to view the AGN from the direction of the scattering medium. The presence of two lines-of-sight allows to constrain the inclination dependence of various emission properties, and in particular the three dimensional velocity field in the BLR. Since the BLR is very likely disk-like, as indicated in radio-loud AGN (e.g., Wills \& Browne 1986; Fine et al. 2011), and generally rotationally supported, the observed line-of-sight velocity dispersion $W$ is inclination dependent. Since the virial black hole mass estimate $M_{\mathrm{BH}} \propto W^{2}$, incli- 


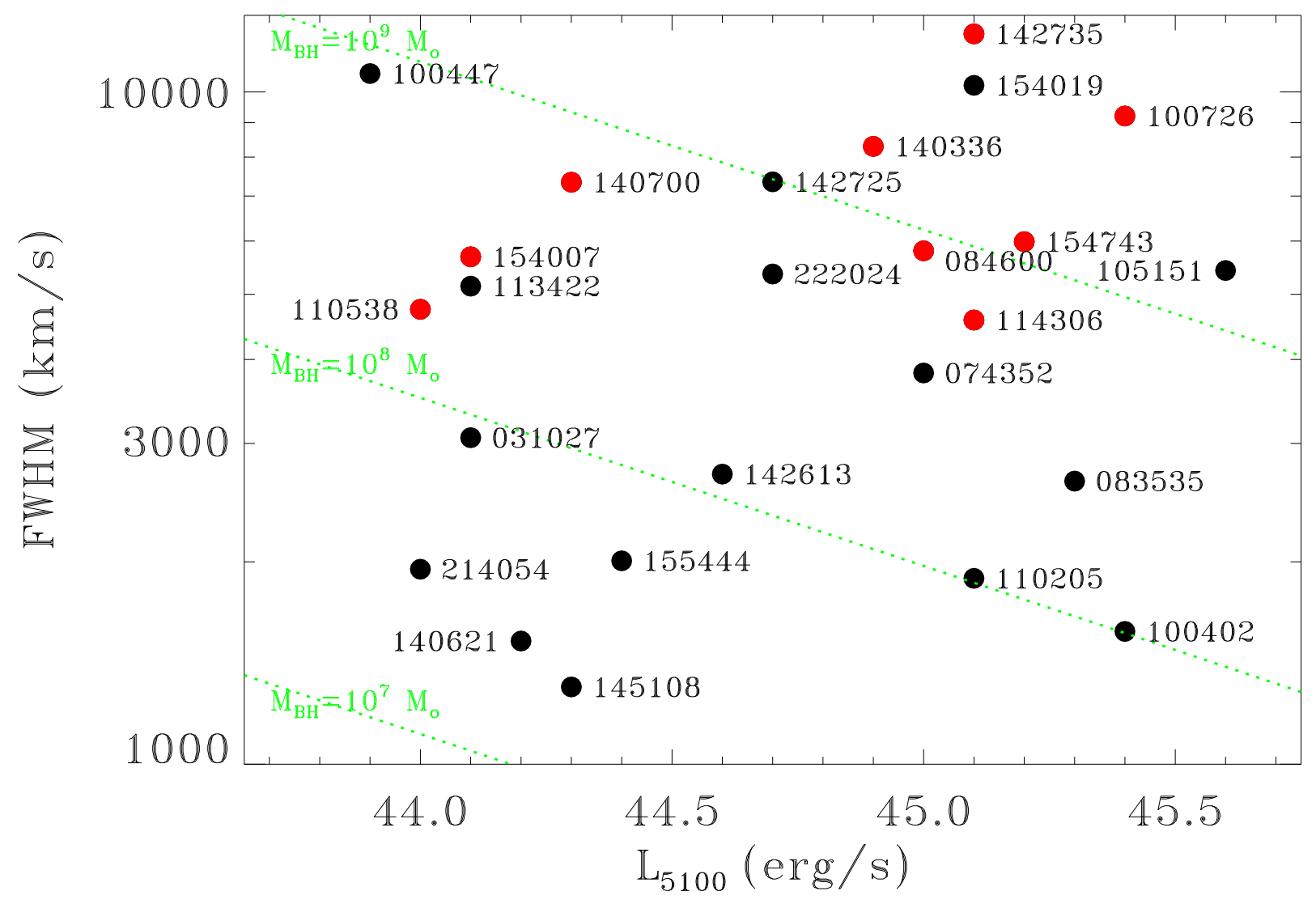

Figure 1. The distribution of the $5100 \AA$ luminosity $L_{5100}$ versus the broad H $\alpha$ FHM of the 25 quasars selected for the VLT spectropolarimetry. The $L_{5100}$ vs. FWHM plain was divided into a (logarithmically) uniform grid, and the sample consists of the brightest SDSS/DR7 quasar in each rectangle, which satisfies $z<0.12$ or $0.21<z<0.38$. This process is repeated for radio quiet QSO (RQQ, black) and for radio loud QSO (RLQ, red). This selection scheme ensures the widest possible coverage in $L_{5100}$, FWHM, and $M_{\mathrm{BH}}$ estimated with the virial method (marked by diagonal dashed lines).

nation effects can lead to a significant bias. In particular, a face-on view and a low $M_{\mathrm{BH}}$ in objects with low $W$ are difficult to tell apart. Spectropolarimetry allows to constrain the inclination angle, and thus potentially reduce significantly the inclination bias in the $M_{\mathrm{BH}}$ estimates. Furthermore, if the scattering region is located in the AGN equatorial plane, it sees the full rotation amplitude of the BLR, and the light reflected (and thence polarized) will show the intrinsic value of the BLR line width.

The accuracy of $M_{\mathrm{BH}}$ estimates may be compromised by additional systematic effects. First of all, the virial method assumes that the BLR is dominated by rotation, while the presence of outflowing material on different scales is a widespread characteristic of AGN. An outflow will increase $W$ and induce a bias for the $M_{\mathrm{BH}}$ estimates. The outflow intensity may be linked to the AGN

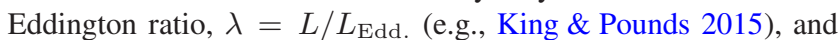
then, ultimately, to $M_{\mathrm{BH}}^{-1}$. The effects of orientation bias are even more complex in case of an outflowing component, as the outflow occurs mainly along the polar direction, which will produce an opposite bias compared to the rotational component.

We recently obtained spectropolarimetric observations with FORS2 at the VLT of the narrow line Seyfert 1 PKS 2004-447 (NLSy1 are type 1 AGN in which the broad-line FWHM are < $2000 \mathrm{~km} / \mathrm{s}$ ) (see Baldi et al. 2016). We recovered all features typical of the equatorial scattering model (ESM, Smith et al. 2005); in particular its polarized spectrum shows two peaks separated by $\sim 9,100$ $\mathrm{km} / \mathrm{s}$. The broadening with respect to the FWHM of $1447 \mathrm{~km} \mathrm{~s}^{-1}$ measured in direct-light (Oshlack et al. 2001) is a dramatic indication that in this source the $M_{\mathrm{BH}}$ value based on the virial method is underestimated by a factor $\sim 40$ (!). PKS $2004-447$ is a very challenging target for spectropolarimetric observations. Indeed, the ESM predicts that the percentage of polarization decreases for objects seen close-to face-on, a condition met by this source as witnessed by its detection (and rapid variability) in $\gamma$-rays by Fermi (Calderone et al. 2011), clear evidence for a relativistic jet oriented at a small angle to the line-of-sight. Nonetheless, despite the extremely low level of polarization $\sim 0.1 \%$, we were able to obtain a robust $M_{\mathrm{BH}}$ estimate.

The purpose of this study is to follow up on the study of Baldi et al. (2016) and derive systematic high signal-to-noise ratio observations of the broad line and continuum spectropolarimetric properties of a representative sample of 25 low redshift $(z<0.37)$ QSO. The QSO are selected to cover uniformly a wide range of luminosities and BLR line widths. Specifically, the sample covers a range in luminosities at $5100 \AA$ of $L_{5100} \sim 10^{44}-10^{46} \mathrm{erg} \mathrm{s}^{-1}$, and $\mathrm{FWHM}=1000-10,000 \mathrm{~km} \mathrm{~s}^{-1}$, which corresponds to black 
Table 1. Sample properties

\begin{tabular}{|c|c|c|c|c|c|c|c|c|c|c|c|c|}
\hline $\begin{array}{l}\text { Name } \\
\text { (1) }\end{array}$ & $\begin{array}{l}\text { Alt. name } \\
\text { (2) }\end{array}$ & $\begin{array}{l}\text { Z } \\
\text { (3) }\end{array}$ & $\begin{array}{l}\mathrm{m}_{r} \\
\text { (4) }\end{array}$ & $\begin{array}{c}\log \mathrm{L} \\
5100 \AA \\
(5)\end{array}$ & $\begin{array}{c}\log L \\
(6)\end{array}$ & $\begin{array}{l}\mathrm{H} \alpha \\
\text { FWHM } \\
\text { (7) }\end{array}$ & $\begin{array}{c}\mathrm{EW} \\
(8)\end{array}$ & $\begin{array}{c}\log \mathrm{M}_{\mathrm{BH}} \\
{\left[\mathrm{M}_{\odot}\right]} \\
(9)\end{array}$ & $\begin{array}{c}\log L / L_{E d d} \\
\text { (10) }\end{array}$ & $\begin{array}{r}\mathrm{R} \\
(11)\end{array}$ & & $\begin{array}{l}\alpha_{\mathrm{opt}} \\
(12)\end{array}$ \\
\hline J031027.83-004950.8 & & 0.080 & 15.81 & 44.1 & 42.6 & 2494 & 246 & 7.93 & -0.97 & $<0.8$ & & -1.09 \\
\hline J074352.02+271239.5 & & 0.252 & 15.71 & 45.0 & 43.9 & 3049 & 509 & 8.59 & -0.69 & 0.4 & & -1.41 \\
\hline J083535.81+245940.2 & PG 0832+251 & 0.329 & 15.93 & 45.3 & 44.2 & 2287 & 485 & 8.41 & -0.23 & 1.0 & & -1.52 \\
\hline J084600.37+070424.7 & & 0.342 & 16.99 & 45.0 & 44.0 & 5325 & 660 & 8.92 & -1.09 & 430.0 & RL & -1.34 \\
\hline $\mathrm{J} 100402.62+285535.4$ & PG 1001+291 & 0.328 & 15.72 & 45.4 & 44.1 & 1660 & 330 & 8.03 & 0.28 & $<0.4$ & & -1.82 \\
\hline $\mathrm{J} 100447.61+144645.6$ & MRK 0715 & 0.084 & 16.35 & 43.9 & 42.5 & 8121 & 179 & 8.94 & -2.13 & 10.0 & & -0.06 \\
\hline $\mathrm{J} 100726.10+124856.2$ & PG 1004+130 & 0.240 & 15.47 & 45.4 & 43.8 & 5996 & 213 & 9.54 & -1.27 & 220.0 & RL & -1.79 \\
\hline J105151.44-005117.7 & PG 1049-005 & 0.359 & 15.69 & 45.6 & 44.4 & 4794 & 439 & 9.17 & -0.72 & $<0.7$ & & -1.55 \\
\hline $\mathrm{J} 110205.92+084435.7$ & & 0.294 & 16.35 & 45.1 & 43.7 & 1692 & 285 & 8.00 & -0.06 & $<1.1$ & & -1.56 \\
\hline$J 110538.99+020257.3$ & & 0.105 & 16.53 & 44.0 & 42.8 & 3734 & 367 & 8.27 & -1.39 & 520.0 & RL & -0.24 \\
\hline $\mathrm{J} 113422.47+041127.7$ & & 0.108 & 16.41 & 44.1 & 42.9 & 3896 & 408 & 8.40 & -1.40 & $<1.1$ & & -0.77 \\
\hline $\mathrm{J} 114306.02+184342.9$ & & 0.374 & 16.90 & 45.1 & 43.9 & 3873 & 506 & 8.79 & -0.80 & 180.0 & RL & -1.37 \\
\hline $\mathrm{J} 140336.43+174136.1$ & & 0.222 & 16.60 & 44.9 & 43.6 & 11004 & 334 & 9.21 & -1.41 & 54.0 & RL & -1.62 \\
\hline $\mathrm{J} 140621.89+222346.5$ & PG $1404+226$ & 0.097 & 16.09 & 44.2 & 42.8 & 1442 & 318 & 7.36 & -0.32 & 1.5 & & -1.57 \\
\hline $\mathrm{J} 140700.40+282714.6$ & MRK 0668 & 0.076 & 15.61 & 44.3 & 43.1 & 5943 & 366 & 8.79 & -1.63 & 580.0 & RL & +0.04 \\
\hline $\mathrm{J} 142613.32+195524.7$ & & 0.213 & 16.67 & 44.6 & 43.3 & 2462 & 386 & 8.06 & -0.62 & $<1.4$ & & -1.16 \\
\hline $\mathrm{J} 142725.05+194952.2$ & MRK 0813 & 0.110 & 15.51 & 44.7 & 43.4 & 6248 & 385 & 8.98 & -1.44 & 0.2 & & -1.42 \\
\hline $\mathrm{J} 142735.61+263214.5$ & PG $1425+267$ & 0.364 & 16.61 & 45.1 & 44.1 & 8226 & 498 & 9.66 & -1.64 & 710.0 & RL & -1.43 \\
\hline $\mathrm{J} 145108.76+270926.9$ & PG $1448+273$ & 0.064 & 15.41 & 44.3 & 42.7 & 1395 & 198 & 7.29 & -0.12 & 0.6 & & -1.27 \\
\hline $\mathrm{J} 154007.84+141137.0$ & & 0.119 & 16.95 & 44.1 & 42.9 & 4496 & 375 & 8.46 & -1.51 & 150.0 & RL & -0.54 \\
\hline $\mathrm{J} 154019.57-020505.4$ & & 0.320 & 16.78 & 45.1 & 43.9 & 9217 & 387 & 9.47 & -1.52 & 4.1 & & -1.20 \\
\hline $\mathrm{J} 154743.54+205216.7$ & PG $1545+210$ & 0.264 & 15.60 & 45.2 & 44.0 & 6828 & 498 & 9.07 & -1.00 & 810.0 & RL & -1.42 \\
\hline $\mathrm{J} 155444.58+082221.5$ & PG $1552+085$ & 0.119 & 15.81 & 44.4 & 43.1 & 1553 & 313 & 7.73 & -0.43 & $<0.9$ & & -1.21 \\
\hline $\mathrm{J} 214054.56+002538.2$ & & 0.083 & 16.45 & 44.0 & 42.5 & 1689 & 212 & 7.49 & -0.63 & 0.8 & & -0.62 \\
\hline $\mathrm{J} 222024.59+010931.3$ & & 0.212 & 16.61 & 44.7 & 43.4 & 5018 & 309 & 8.73 & -1.14 & $<1.7$ & & -0.89 \\
\hline
\end{tabular}

Column description: 1) name, 2) alternative name, 3) redshift, 4) $r$ band SDSS magnitude, 5) logarithm of the luminosity at $5100 \AA$ in $\mathrm{erg} \mathrm{s}^{-1}$ (the values from column 5 to 10 are from Shen et al. 2011), 6 - 8) luminosity ( $\mathrm{erg} \mathrm{s}^{-1}$ ), full width at half maximum (FWHM, $\mathrm{km} \mathrm{s}^{-1}$ ), and equivalent width (EW, $\AA$ ) of the broad $\mathrm{H} \alpha$ line, 9) logarithm of the black hole mass in solar masses, estimated with the virial method, 10) logarithm of the bolometric luminosity with respect to the Eddington luminosity, 11) radio loudness parameter (we also indicate with RL the radio-loud sources), 12) slope of the optical continuum, $I_{\lambda}$.

hole masses (estimated with the virial method) of $M_{\mathrm{BH}} \sim 2 \times 10^{7}$ to $5 \times 10^{9} M_{\odot}$.

We use the spectropolarimetry to explore the BLR structure and to study the implications for the measurements of the black hole masses. The plan of the paper is as follows: in Sect. 2 we describe the sample selection; the observations and data analysis are discussed in Sect. 3; we then estimate the integrated (Sect. 4) and spectrally resolved (Sect. 5) polarization parameters of the BLR. In Sect. 6 we used our observations to explore the polarization mechanism in QSOs. The results are discussed in Sect. 7, while in Sect. 8 we give a summary of our findings and draw our conclusions. A cosmology with $H_{0}=72 \mathrm{~km} \mathrm{~s}^{-1} \mathrm{Mpc}^{-1}, \Omega_{m}=0.30$, and $\Omega_{\Lambda}=0.70$ is assumed.

\section{SAMPLE SELECTION}

The targets have been selected from the SDSS/DR7 catalog of QSOs produced by Schneider et al. (2010) and analyzed by Shen et al. (2011). For low redshift QSOs the best suited permitted line for an optical study is $\mathrm{H} \alpha$. The high accuracy needed for the spectropolarimetric analysis requires to avoid the main telluric absorption features (namely the $\mathrm{O}_{2}$ bands at $\lambda \sim 7600 \AA$ and the $\mathrm{H}_{2} \mathrm{O}$ absorptions at $\lambda \gtrsim 9300 \AA$ ) in the region of interest, $\sim 10,000$ $\mathrm{km} / \mathrm{s}$ on each side of $\mathrm{H} \alpha$, leading to the following redshift constraints: $z<0.12$ and $0.21<z<0.38$. The need for a high $\mathrm{S} / \mathrm{N}$ requires to focus on bright targets and we adopted as an initial threshold $r<17$. When combined with a declination $\delta<30^{\circ}$ to ensure VLT observability, these constraints select 128 QSOs.

The final selection aims at exploring the properties of QSOs across the widest possible range of luminosities and estimated black hole masses. We located the 128 QSOs selected above in a diagram comparing the $L_{5100}$ optical luminosity and the FWHM of the broad $\mathrm{H} \alpha$ line. We then divided this diagram with a (logarithmically) uniform grid and within each rectangle we picked the source with the highest optical flux to further improve the data quality (as a result the median flux of the selected sources is $\sim 8 \times 10^{-16} \mathrm{erg}$ $\mathrm{s}^{-1} \AA^{-1} \mathrm{~cm}^{-2}$, about twice the selection threshold).

Since the properties of radio loud and radio quiet QSOs (RLQs and RQQs) might differ, we made separate selections of QSOs belonging to the two sub-classes. Shen et al. (2011) lists the radioloudness parameter $R$, i.e., the ratio of the flux density at rest frame $6 \mathrm{~cm}$ and $2500 \AA$, estimated following the method of Jiang et al. (2007): the rest frame $6 \mathrm{~cm}$ data are obtained by extrapolating the Faint Images of the Radio Sky at Twenty centimeters survey (FIRST, Becker et al. 1995; Helfand et al. 2015) 1.4 GHz measurements assuming a power-law slope of 0.5 , while the $2500 \AA$ flux density is extrapolated from a power-law fit to the SDSS broadband photometry. A significant issue related to this definition is the presence, revealed by the visual inspection of the FIRST images of the selected QSOs, of several objects with radio structures extending well beyond the $30^{\prime \prime}$ radius they adopted as the limit to include a radio component into the total radio emission (see Sect. 4.3). For this reason we preferred to define the radio-loudness pa- 

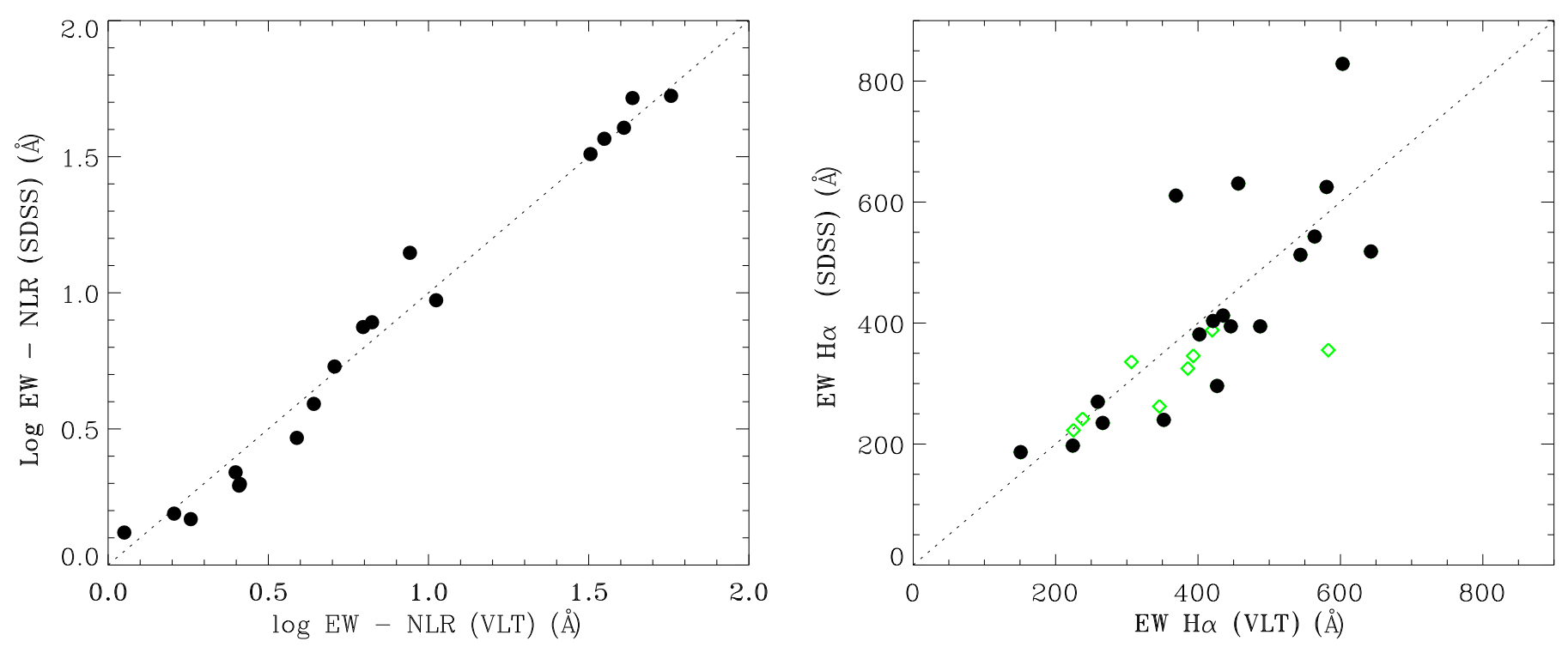

Figure 2. Comparison of the SDSS and VLT measurements of the EW of the forbidden narrow lines (left) and the broad H $\alpha$ (right). The green diamonds in the right panel mark the sources where the narrow line fluxes could not be measured. These measurements were used to obtain an absolute flux calibration for the VLT spectra from the SDSS spectra. The left panel indicates generally small variability in both the continuum and BLR.

Table 2. Observations log.

\begin{tabular}{lcccc}
\hline Name & Date & Obs. time & Cal. & S/N \\
\hline J031027.83-004950.8 & $2016-09-18$ & 41 & 0.96 & 267 \\
J074352.02+271239.5 & $2016-11-24$ & 41 & 1.05 & 408 \\
J083535.81+245940.2 & $2016-12-20$ & 15 & 0.89 & 139 \\
J084600.37+070424.7 & $2016-12-04$ & 41 & 1.04 & 205 \\
J100402.62+285535.4 & $2016-12-16$ & 41 & & 291 \\
J100447.61+144645.6 & $2016-12-22$ & 41 & 1.20 & 330 \\
J100726.10+124856.2 & $2016-12-23$ & 41 & 1.17 & 357 \\
J105151.44-005117.7 & $2017-01-11$ & 41 & 0.93 & 202 \\
J110205.92+084435.7 & $2016-12-23$ & 41 & & 226 \\
J110538.99+020257.3 & $2017-01-04$ & 41 & 0.81 & 298 \\
J113422.47+041127.7 & $2017-01-10$ & 41 & 1.17 & 340 \\
J114306.02+184342.9 & $2017-01-10$ & 41 & 0.99 & 155 \\
J140336.43+174136.1 & $2017-03-03$ & 41 & & 308 \\
J140621.89+222346.5 & $2017-03-07$ & 56 & & 277 \\
J140700.40+282714.6 & $2017-02-09$ & 41 & 0.89 & 345 \\
& $2017-03-06$ & 41 & 0.76 & 413 \\
J142613.32+195524.7 & $2017-03-07$ & 41 & & 233 \\
J142725.05+194952.2 & $2017-03-12$ & 54 & & 361 \\
J142735.61+263214.5 & $2017-03-03$ & 41 & 1.20 & 185 \\
J145108.76+270926.9 & $2017-03-06$ & 41 & 0.88 & 593 \\
J154007.84+141137.0 & $2017-03-13$ & 41 & 0.77 & 219 \\
J154019.57-020505.4 & $2017-02-10$ & 41 & 1.60 & 161 \\
J154743.54+205216.7 & $2017-03-12$ & 41 & 1.01 & 327 \\
J155444.58+082221.5 & $2017-03-14$ & 33 & & 298 \\
J214054.56+002538.2 & $2016-09-18$ & 41 & 0.76 & 265 \\
J222024.59+010931.3 & $2016-09-18$ & 41 & & 238 \\
\hline
\end{tabular}

Column description: 1) source name, 2) date of observations, 3) exposure time in minutes, 4) ratio between the VLT and SDSS continuum flux, 5) S/N per resolution element at $6750 \AA$.

rameter (see Table 1) by including the radio flux densities of all the NVSS (National Radio Astronomy Observatory Very Large Array Sky Survey, Condon et al. 1998) components (because this survey is more sensitive to diffuse radio structure compared to FIRST) as- sociated with each QSO. For the sources not detected by the NVSS, we used either the FIRST detection or upper limit.

The final sample consists of 16 RQQs and 9 RLQs (see Figure 1 ), respectively. RLQs only populate the upper part of the diagram because there are no such objects, among the 128 selected initially, with a FWHM smaller than $3000 \mathrm{~km} \mathrm{~s}^{-1}$.

The main properties of the 25 QSOs of the sample, extracted from the measurements obtained by Shen et al. (2011), are given in Table 1. As a result of our selection method we have objects spanning a factor $\sim 100$ in luminosity $\left(\log L_{5100}=43.9-45.6\right.$ in erg s ${ }^{-1}$ units), and with a broad $\mathrm{H} \alpha$ width from $\sim 1400$ to $\sim$ $11,000 \mathrm{~km} \mathrm{~s}^{-1}$, resulting in black hole masses (estimated with the virial method) ranging from $2 \times 10^{7}$ to $5 \times 10^{9} M_{\odot}$.

\section{OBSERVATIONS AND DATA ANALYSIS}

The selected 25 sources were observed in spectropolarimetric mode with the FORS2 mounted at the UT1 telescope of the 8.2-m ESO Very Large Telescope (VLT), program 098.B-0234(A), each source fitting into a single 1 hour observing block. The log of the observations is presented in Table 2.

The observations were obtained with the GRISM-300I, providing a spectral resolution of $R \sim 660$ at $8600 \AA$, used in combination with the blocking filter OG590. The usable wavelength range is $6100-9300 \AA$. The $1^{\prime \prime}$ wide slit was oriented along the parallactic angle and the multi-object spectropolarimetry (PMOS) observation was performed with a $2048 \times 2048$ pixel CCD with a spatial resolution of $0.126^{\prime \prime} /$ pixel.

The measurements at two retarder angles are sufficient to calculate the linear Stokes parameters. However, in order to reduce instrumental issues (Patat \& Romaniello 2006) we used four halfwave plate angles $(0,22.5,45$ and 67.5 degrees) for our observations. For each source we then obtained four spectra (split in two exposures for cosmic rays removal), with the half-plate oriented at the four position angles. The observing time per source is generally $\sim 41$ minutes, with a few exceptions due, e.g., to saturation limits or scheduling problems, see Table 2. One source was observed twice. 


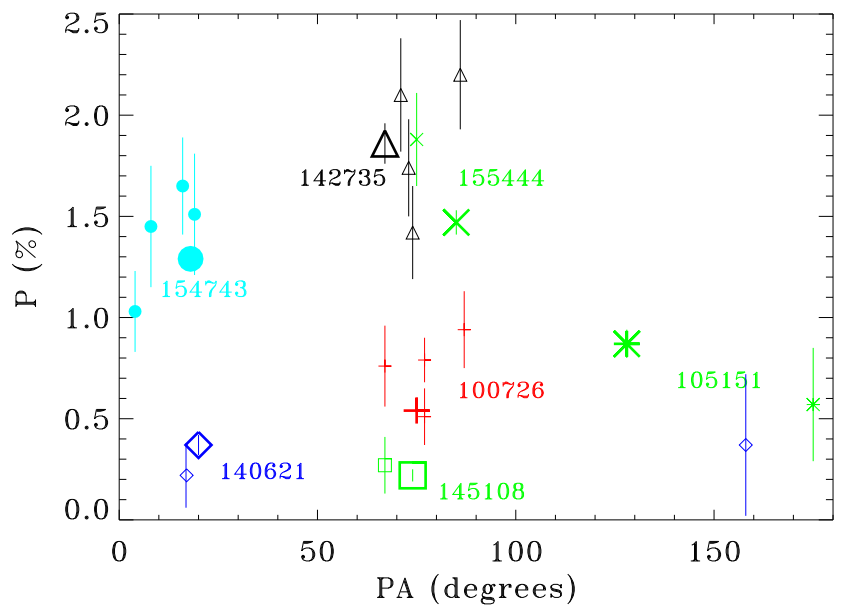

Figure 3. Comparison of $P(\%)$ and $\mathrm{PA}($ deg.) measured here with the white light measurements of Berriman et al. (1990) for the seven sources in common. Each object is marked with a different symbol. The large symbol is the VLT measurement. For clarity we reproduce only the errors for $P$. The differences are generally consistent with the measurement errors. The polarization appears to be stable over $\sim 30-40$ years.

The data were analyzed by using the standard VLT pipeline with the ESO Reflex workflow (Hook et al. 2008). The frames were first bias-subtracted and then flat-fielded using lamp flats. After removing cosmic ray events, the different exposures for the four halfwave plate positions were combined for each observing block. Onedimensional spectra, with identical aperture widths for the ordinary (o) and extraordinary (e) rays for each of the four half-wave plate orientations, were then extracted (from 12 pixels corresponding to $\left.1.5^{\prime \prime}\right)$. The wavelength calibration was performed using standard arc lamps. The o- and e-rays were resampled to the same linear spectral dispersion and combined to determine the Stokes parameters I, Q, and $\mathrm{U}$ (as well as the associated uncertainties) using the procedures described by Cohen et al. (1995) and Vernet et al. (2001). The $\mathrm{S} / \mathrm{N}$ in the total spectrum in each spectral element, estimated at $6750 \AA$, ranges from $\sim 140$ to $\sim 600$ with a median value of $\sim 300$ (see Table 2). During the observations the seeing varied between 0. ' 36 and 1 ". 20 , with a median value of 0. ' 60 .

To maximize the efficiency of the observing program we did not obtain observations of standard stars. Nonetheless, a comparison of the SDSS and VLT spectra can be used to derive a relative flux calibration. We estimated the ratio of the raw VLT and the calibrated SDSS spectra for each source. The shape of this ratio is very similar for all sources, reproducing mainly the changes of efficiency with wavelength of the VLT spectropolarimetric observations. The curve representing the median ratio versus wavelength can be well reproduced with a quadratic curve over the whole spectral range, with residuals never exceeding $3 \%$. We will use this curve to correct the VLT spectra and to obtain their relative flux calibration.

We obtained also an absolute calibration for our spectra by measuring the equivalent width (EW) of the forbidden narrow lines. By assuming that their fluxes do not change between the epoch of the SDSS (2001 - 2008) and VLT spectra, any variation of their EW can be interpreted as the result of continuum flux variability. The EW comparison is possible for the 17 sources (with two measurements for J140700) for which we could obtain an accurate (with an error smaller than $\sim 20 \%$ ) EW measurement of either the [O III] $\lambda 5007$ line or [S II] $\lambda 6716,6731$ doublet, depending on the target redshift. The result is shown in Figure 2 (left panel). The SDSS and VLT values for the EW agree, in all but one case, to within $30 \%$. The resulting variation of the continuum flux (ranging from 0.76 to 1.60 ) from the SDSS to the VLT data is reported in Table 2.

The EW of the broad $\mathrm{H} \alpha$ line show changes of similar amplitude, see Figure 2 (right panel). We can extend this comparison also to the objects lacking measurable narrow lines (green diamonds in the figure). For these 8 objects the EW changes are of comparable (or even smaller) amplitude. We conclude that in all of the 25 QSOs observed there were no large changes in the last 10-15 years in the relative fluxes of the continuum and the broad $\mathrm{H} \alpha$ line, and in at least 17 of the QSOs the absolute continuum and BLR fluxes also did not show large changes.

\section{INTEGRATED POLARIZATION MEASUREMENTS}

From the $I, Q$, and $U$ Stokes parameters we computed the polarized spectrum $I_{P}=\left(Q^{2}+U^{2}\right)^{1 / 2}$, the corresponding degree of polarization $P=I_{P} / I$, and the polarization position angle, $\mathrm{PA}$ (measured counterclockwise starting from North). We started the analysis by deriving integrated polarization measurements for the BLR and the continuum emission. The continuum polarization was estimated by considering two spectral regions between 150 and $300 \AA$ wide flanking the broad $\mathrm{H} \alpha$ line. The width and location of the region of integration was selected, on an object-by-object basis (see the spectra presented in the Supplementary Material), in order to avoid telluric absorption bands and, in some cases, the narrow emission lines [O I] $\lambda \lambda 6300,6363$ or [S II] $\lambda \lambda 6716,6731$. The Stokes parameters measured on the blue and red side were linearly interpolated to obtain their values at the rest frame $\mathrm{H} \alpha$ position. All measurements of the polarized flux are at a significance higher that $2 \sigma$, except for $\mathrm{J} 110538$ (see Table 3).

Seven of the sources of our sample are in common with those studied by Berriman et al. (1990): they obtained white light polarization of the 114 PG QSOs between 1977 and 1986. In Figure 3 we compare their results with the continuum polarization from the VLT data. There is close agreement between these measurements, with changes overall consistent within the errors. Thus, the polarization of these QSOs appears to be stable over $\sim 30-40$ years.

We measured the BLR polarization integrating over a spectral range spanning twice the $\mathrm{H} \alpha \mathrm{FWHM}$ of each given source. In order to isolate the intrinsic BLR polarization we removed the underlying continuum polarization: the linear fit to the continuum $I, Q$, and $U$ derived above was subtracted from the data before deriving the polarization parameters. The measurements of the BLR polarized flux are at a significance higher that $2 \sigma$, in all but two sources. In addition, in J145108 the BLR polarization measurements are compromised by the presence of a cosmic ray that is not effectively removed by the reduction pipeline.

\subsection{Dilution from unpolarized emission}

The presence of unpolarized emission might affect the measurements of the intrinsic nuclear polarization. In particular, the host emission dilutes the continuum polarization, while the narrow lines (i.e., the $[\mathrm{N} \mathrm{II}]$ doublet and the narrow $\mathrm{H} \alpha$ ) might contaminate the BLR polarization. We stress, however, that the presence of unpolarized components only affects the percentage of polarization, while the polarized flux and the polarization angle are not affected. 
Table 3. Polarization measurements

\begin{tabular}{|c|c|c|c|c|c|c|c|c|c|c|}
\hline $\begin{array}{l}\text { Name } \\
\text { (1) }\end{array}$ & $\begin{array}{l}P \text { cont. }(\%) \\
\text { (2) }\end{array}$ & $\begin{array}{r}\text { PA cont. } \\
\text { (3) }\end{array}$ & & $\begin{array}{l}P \text { BLR (\%) } \\
\text { (4) }\end{array}$ & $\begin{array}{r}\text { aver. } P \text { BLR (\%) } \\
(5)\end{array}$ & $\begin{array}{r}\text { PA BLR } \\
(6)\end{array}$ & $\begin{array}{r}\text { PA swing } \\
(7)\end{array}$ & $\begin{array}{r}\text { W (I) } \\
(8)\end{array}$ & $\begin{array}{r}\mathrm{W}\left(\mathrm{I}_{P}\right) \\
(9)\end{array}$ & $\begin{array}{r}I_{P}-I \text { shift } \\
(10)\end{array}$ \\
\hline J031027 & $0.18 \pm 0.06$ & $68 \pm 9$ & & $0.06 \pm 0.06$ & $0.35(0.15)$ & - & $78 \pm 11$ & 1974 & $3890_{-650}^{+500}$ & $+670 \pm 510$ \\
\hline J074352 & $0.47 \pm 0.03$ & $59 \pm 2$ & & $0.24 \pm 0.03$ & $0.27(0.20)$ & $68 \pm 4$ & - & 2148 & $5020_{-450}^{+320}$ & $+880 \pm 440$ \\
\hline J083535 & $1.71 \pm 0.09$ & $80 \pm 2$ & & $0.05 \pm 0.07$ & $0.26(0.13)$ & - & - & 2239 & $4120_{-790}^{+720}$ & $+740 \pm 420$ \\
\hline J084600 & $1.23 \pm 0.07$ & $67 \pm 2$ & & $0.36 \pm 0.05$ & $0.32(0.15)$ & $67 \pm 3$ & $30 \pm 7$ & 3186 & $3940_{-480}^{+550}$ & $-200 \pm 430$ \\
\hline $\mathrm{J} 100402$ & $0.36 \pm 0.05$ & $18 \pm 4$ & & $0.13 \pm 0.05$ & $0.13(0.07)$ & $23 \pm 10$ & - & 1773 & $4220_{-730}^{+950}$ & $+890 \pm 580$ \\
\hline J100447 & $0.36 \pm 0.06$ & $35 \pm 5$ & & $0.27 \pm 0.07$ & $0.15(0.17)$ & $83 \pm 7$ & - & 3551 & $4110_{-870}^{+930}$ & $-290 \pm 520$ \\
\hline J100726 & $0.54 \pm 0.06$ & $75 \pm 3$ & & $0.22 \pm 0.09$ & $0.21(0.11)$ & $73 \pm 11$ & - & 4603 & $5860_{-1040}^{+1670}$ & $+1680 \pm 810$ \\
\hline $\mathrm{J} 105151$ & $0.87 \pm 0.07$ & $128 \pm 2$ & & $0.07 \pm 0.05$ & $0.38(0.14)$ & - & $79 \pm 9$ & 3222 & $5230_{-610}^{+1040}$ & $-210 \pm 400$ \\
\hline J110205 & $0.14 \pm 0.07$ & $50 \pm 14$ & & $0.25 \pm 0.07$ & $0.24(0.12)$ & $29 \pm 7$ & - & 1618 & $2960_{-590}^{+740}$ & $+300 \pm 260$ \\
\hline J110538 & $0.03 \pm 0.07$ & - & & $0.10 \pm 0.05$ & $0.14(0.07)$ & $111 \pm 14$ & - & 2523 & $5170_{-1010}^{+1390}$ & $-470 \pm 620$ \\
\hline J113422 & $0.59 \pm 0.05$ & $72 \pm 2$ & & $0.17 \pm 0.04$ & $0.17(0.09)$ & $56 \pm 7$ & - & 2710 & $4460_{-770}^{+1610}$ & $+30 \pm 530$ \\
\hline J114306 & $0.21 \pm 0.10$ & $28 \pm 14$ & & $0.32 \pm 0.07$ & $0.32(0.27)$ & $45 \pm 6$ & - & 2422 & $3460_{-610}^{+980}$ & $-500 \pm 350$ \\
\hline J140336 & $1.02 \pm 0.05$ & $132 \pm 1$ & & $0.76 \pm 0.06$ & $0.36(0.26)$ & $84 \pm 2$ & $19 \pm 7$ & 4808 & $4380_{-360}^{+420}$ & $+2050 \pm 310$ \\
\hline J140621 & $0.39 \pm 0.05$ & $19 \pm 4$ & & $0.35 \pm 0.05$ & $0.40(0.25)$ & $9 \pm 4$ & - & 1225 & $\begin{array}{l}1870_{-300}^{+500} \\
\end{array}$ & $+20 \pm 70$ \\
\hline $\mathrm{J} 140700$ (a) & $0.79 \pm 0.07$ & $136 \pm 2$ & & $0.48 \pm 0.05$ & $0.56(0.12)$ & $75 \pm 2$ & $52 \pm 4$ & 4123 & $5690_{-370}^{+380}$ & $-40 \pm 460$ \\
\hline $\mathrm{J} 140700$ (b) & $1.03 \pm 0.06$ & $136 \pm 2$ & & $0.56 \pm 0.04$ & $0.50(0.14)$ & $64 \pm 2$ & $41 \pm 5$ & 4255 & $5700_{-480}^{+430}$ & $-570 \pm 220$ \\
\hline $\mathrm{J} 142613$ & $0.18 \pm 0.07$ & $125 \pm 10$ & & $0.19 \pm 0.06$ & $0.29(0.15)$ & $20 \pm 8$ & - & 2020 & $\begin{array}{l}4740_{-770}^{+570} \\
\end{array}$ & $-200 \pm 450$ \\
\hline J142725 & $0.84 \pm 0.05$ & $70 \pm 2$ & & $0.52 \pm 0.04$ & $0.36(0.13)$ & $77 \pm 2$ & $22 \pm 4$ & 3515 & $4750_{-490}^{+1010}$ & $+550 \pm 260$ \\
\hline J142735 & $1.86 \pm 0.10$ & $67 \pm 2$ & & $0.37 \pm 0.05$ & $0.37(0.29)$ & $44 \pm 3$ & $14 \pm 9$ & 4982 & $5410_{-450}^{+500}$ & $+1820 \pm 620$ \\
\hline $\mathrm{J} 145108$ & $0.23 \pm 0.03$ & $73 \pm 3$ & & - & $0.40(0.20)$ & - & - & 1261 & - & - \\
\hline $\mathrm{J} 154007$ & $0.72 \pm 0.09$ & $48 \pm 4$ & & $0.43 \pm 0.06$ & $0.35(0.09)$ & $56 \pm 3$ & $13 \pm 7$ & 3003 & $3870_{-610}^{+940}$ & $+1030 \pm 360$ \\
\hline J154019 & $2.03 \pm 0.26$ & $89 \pm 4$ & $*$ & $1.30 \pm 0.08$ & $0.77(0.25)$ & $80 \pm 1$ & $12 \pm 4$ & 4978 & $5220_{-510}^{+330}$ & $-500 \pm 300$ \\
\hline $\mathrm{J} 154743$ & $1.29 \pm 0.06$ & $18 \pm 1$ & & $0.28 \pm 0.04$ & $0.20(0.11)$ & $66 \pm 3$ & $5 \pm 8$ & 3186 & $3730_{-670}^{+650}$ & $-160 \pm 350$ \\
\hline $\mathrm{J} 155444$ & $1.23 \pm 0.09$ & $86 \pm 2$ & $*$ & $1.14 \pm 0.06$ & $0.82(0.22)$ & $71 \pm 1$ & $4 \pm 3$ & 1252 & $\begin{array}{l}1480_{-160}^{+230} \\
\end{array}$ & $+100 \pm 70$ \\
\hline $\mathrm{J} 214054$ & $0.67 \pm 0.12$ & $131 \pm 5$ & $*$ & $0.27 \pm 0.06$ & $0.36(0.14)$ & $137 \pm 6$ & - & 1325 & $2990_{-590}^{+1430}$ & $+20 \pm 280$ \\
\hline J222024 & $0.72 \pm 0.07$ & $21 \pm 3$ & & $0.36 \pm 0.06$ & $0.58(0.30)$ & $30 \pm 4$ & $58 \pm 5$ & 2719 & $4250_{-400}^{+540}$ & $+230 \pm 550$ \\
\hline
\end{tabular}

Column description: 1) source name, 2) and 3) percentage of polarization and polarization position angle of the continuum at $6563 \AA$. For the three sources marked with an* we corrected the continuum for the interstella polarization. 4) and 5) integrated $P$ and averaged $P$ (see Section 5 for its definition) of the BLR over twice the width of the broad $\mathrm{H} \alpha$ line after continuum subtraction, 6) PA of the BLR 7) PA swing between the blue and red wing of the BLR, 8) $W(I)$ : interpercentile width (between $25 \%$ and $75 \%$ ) of the BLR in total intensity in $\mathrm{km} \mathrm{s}^{-1}$ and 9) in polarized light $\left(W\left(I_{P}\right)\right.$ ), 10) shift between the line centroid in $I$ and $I_{P}$ in $\mathrm{km} \mathrm{s}^{-1}$.
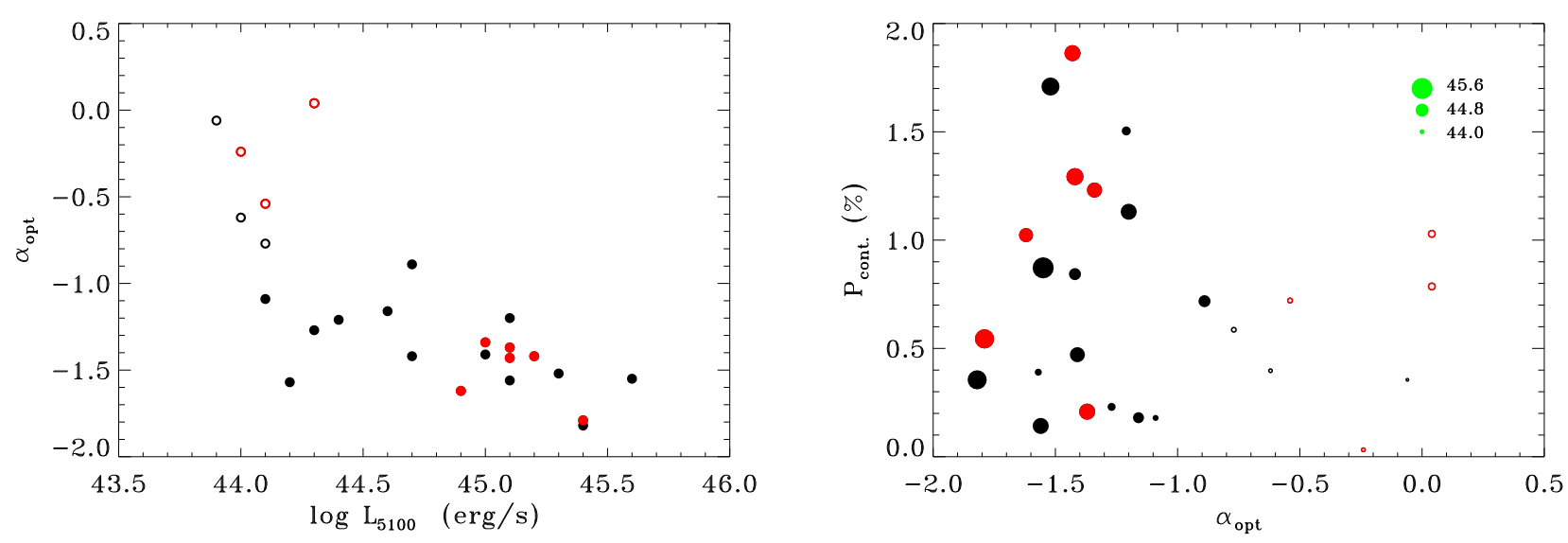

Figure 4. Left: The optical slope $\alpha_{\mathrm{opt}}$ from SDSS versus $\log L_{5100}$. Red dots mark RLQs. All luminous QSOs are blue, and all red QSOs are low luminosity, indicating substantial host contribution. We therefore designate here and below all objects at $\log L_{5100}<44.5$ and $\alpha_{\text {opt }}>-1$ with empty circles. Right: The value of $P_{\text {cont }}(\%)$ versus $\alpha_{\text {opt }}$; the symbol sizes increases with the value of $L_{5100}$ (see legend in the top right corner). The red low luminosity QSOs extend to a lower maximal polarization than the blue high luminosity ones, probably due to the host dilution. Note that the two data points at $\alpha_{\mathrm{opt}}>0$ refer to the two observations of the same object, J140700 (see Table 3). 

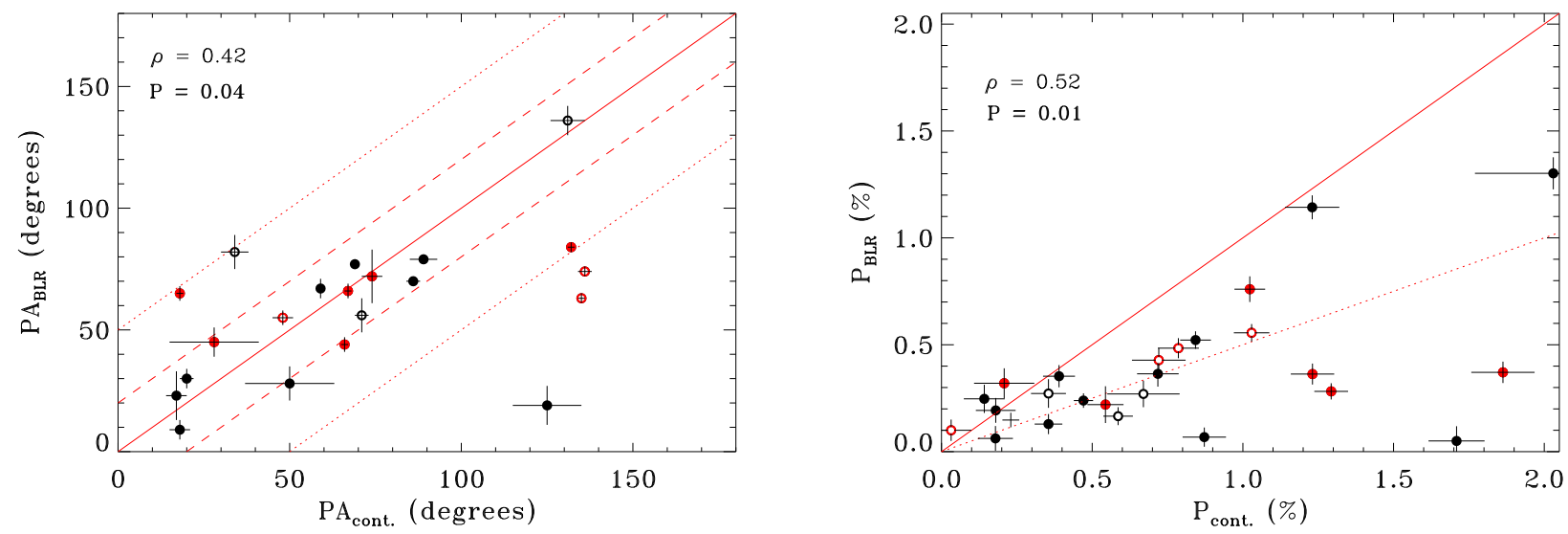

Figure 5. Left: a comparison of the polarization PA of the continuum and of the BLR. The dashed (dotted) lines correspond to a difference of $\pm 20^{\circ}\left( \pm 50^{\circ}\right)$. The two angles differ by less than $20^{\circ}$ in $15 / 20$ objects, and only in $2 / 20$ by more than $50^{\circ}$. This result indicates that in most objects the polarization of the continuum and the BLR are both produced by a single scattering medium. The Spearman rank test indicates a positive correlation between these two quantities with $\rho=0.42$ and a chance probability of 0.04. Right: a comparison of the percentage of polarization of the continuum and of the BLR. The continuum polarization is on average 1.7 times larger than the BLR polarization. The rank test returns $\rho=0.52$ and $P=0.01$. The low luminosity red QSOs, with $\alpha_{\mathrm{opt}}>-1$ and $\log L_{5100}<44.5$, are represented by empty circles (see Figure 4 ). The red symbols are the RLQs. In essentially all objects $P_{\mathrm{BLR}} \leqslant P_{\text {cont }}$. This result suggests that the scattering medium is physically close to the BLR, leading to a significant geometrical cancellation.

\subsubsection{Polarization dilution by the host galaxies}

Analysis of type I AGN SDSS spectra by Stern \& Laor (2012) yields that at the SDSS aperture the host dominates the AGN flux at $7000 \AA$, on average, for luminosities $\log L_{\mathrm{bol}} \leqslant 45$, or equivalently $\log L_{5100} \leqslant 44$, which holds for 3 of the objects in our sample. However, there is still a significant contribution (host/AGN $>30 \%$ ) at $\log L_{5100}<45$, which holds for all of the 11 low redshift $(z<0.12)$ objects in our sample.

In order to explore this issue more quantitatively we measured the power-law slope $\alpha_{\text {opt }}(=d \ln I(\lambda) / d \ln \lambda)$ of the continuum in the SDSS spectra. While the higher luminosity sources have generally blue spectra $\alpha_{\text {opt }} \sim-1.5$, several low luminosity QSOs are quite red (see Figure 4, left panel), suggestive of a significant host contribution.

The presence of the host manifests its effects also on the continuum polarization (see Figure 4, right panel): while the QSO with bluer spectra (and of higher luminosity) show a polarization up to $\sim 2 \%$, the reddest (and least luminous) ones only reach $\sim 1 \%$. This effect is likely caused by the dilution of the unpolarized starlight. Due to the relatively large spread of the distribution of the optical slopes, a correction to derive the genuine nuclear polarization on an object-by-object basis is not deemed to be sufficiently accurate. Furthermore, the extraction region used for the VLT spectra $\left(1^{\prime \prime} \times 1^{\prime \prime} .5\right)$ is significantly smaller than the SDSS fiber $\left(3^{\prime \prime}\right.$ in diameter) and thence the host contribution in the spectropolarimetry data is reduced with respect to the SDSS spectra. In order to highlight possible dilution effects we mark below the six red, low luminosity QSOs $\left(\alpha_{\mathrm{opt}}>-1, \log L_{5100}<44.5\right)$ when relevant for our analysis.

\subsubsection{Polarization dilution from the narrow lines}

The presence of narrow emission lines within the spectral region of integration might reduce the BLR polarization with respect to the continuum. The narrow line region (NLR) extends to a much larger scale than the continuum and the BLR and its emission generally
Table 4. Optical polarization and radio axis of the RL QSOs.

\begin{tabular}{lrrrr}
\hline Name & $P A$ cont. & $P A$ BLR & Radio & Core dominance \\
\hline J084600 & $67 \pm 2$ & $67 \pm 3$ & 82 & 0.35 \\
J100726 & $75 \pm 3$ & $73 \pm 11$ & 111 & 0.01 \\
J110538 & - & $111 \pm 14$ & 150 & 0.64 \\
J114306 & $28 \pm 14$ & $45 \pm 6$ & 24 & 0.70 \\
J140336 & $132 \pm 1$ & $84 \pm 2$ & 156 & 0.20 \\
J140700 & $136 \pm 2$ & $70 \pm 2$ & unres. & - \\
J142735 & $67 \pm 2$ & $44 \pm 3$ & 56 & 0.12 \\
J154007 & $48 \pm 4$ & $56 \pm 3$ & 38 & 0.38 \\
J154743 & $18 \pm 1$ & $66 \pm 3$ & 20 & 0.01 \\
\hline
\end{tabular}

shows a very low level of polarization (e.g., Smith et al. 2002). As already mentioned, the resolution of the VLT data we are using $(\sim$ $450 \mathrm{~km} \mathrm{~s}^{-1}$ ) is not sufficient to properly measure the NLR polarization. We can nonetheless address this issue by considering the SDSS spectra which we used to decompose the different emission lines and to estimate their EWs. The EW values span a large range, but they are all generally smaller than $\sim 10 \AA$.

By assuming that the NLR emission is unpolarized the percentage of polarization of the BLR is reduced with respect to its intrinsic value by a factor $1+E W_{\mathrm{NLR}} / E W_{\mathrm{BLR}}$. For the sources considered the $E W_{\mathrm{BLR}}$ ranges from 300 to $900 \AA$ and thence the NLR effects on the integrated BLR polarization measurements are negligible.

Nonetheless, the NLR might affect the polarization within individual bins in the spectra shown in the Supplementary Material, particularly those corresponding to the $\mathrm{H} \alpha$ and [N II] doublet. However, as already noted above, the only effect is a reduced value of the percentage of polarization.

\subsection{The effects of interstellar polarization}

Dust grains aligned by magnetic fields in our Galaxy are known to polarize optical light (e.g., Mathewson \& Ford 1970) and it is 


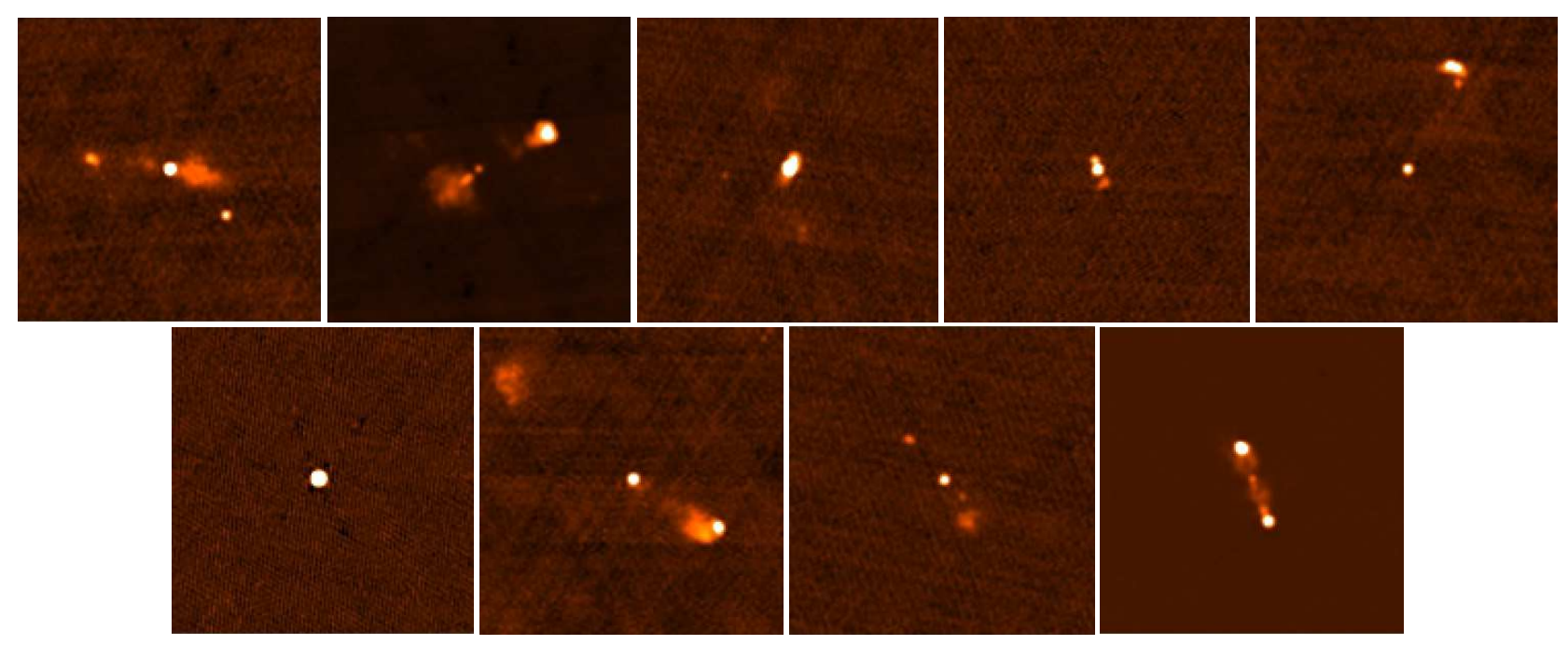

Figure 6. FIRST images of the nine radio-loud QSO, all but one are extended radio sources, reaching sizes of $\sim 1 \mathrm{Mpc}$. Top, from left to right: J084600, J100726, J110538, J114306, and J140336. Bottom: J140700, J142735, J154007, and J154743. The fields of view are 4.5' $\times 4.5^{\prime}$. All objects, except J140700, are resolved, which allows to measure the radio axis and the core dominance. J140700 remains unresolved on VLBA scale, and is a GHz-peaked source (de Vries et al. 1997), which indicates it is a physically compact source, rather than highly beamed.

important to assess the effects of interstellar polarization (ISP) on the QSO properties, in particular on the continuum polarization. In fact, our estimates of the BLR polarization are not affected by the ISP because, by subtracting the continuum polarization, we effectively removed also the ISP contribution.

In the Appendix A, we estimate the ISP for our sources based on the polarization maps produced by the Planck satellite. We conclude that the effects of polarization induced by the interstellar medium have generally a negligible effect on the QSOs polarization properties. We applied the ISP correction only to three sources (namely J154019, J155444, and J214054) where the observed and corrected percentage of polarization differ by more than $2 \sigma$.

\subsection{Continuum vs. BLR polarization}

In Figure 5, left panel, we compare the continuum polarization percentage and position angle, interpolated to $6563 \AA$, with the BLR polarization. Recall that, in order to isolate the net BLR polarization, we derived its polarization parameters after subtraction of the underlying continuum polarization in the Stokes parameters space.

The polarization PA of the continuum and of the BLR differ, in most sources, by less than $30^{\circ}$. More quantitatively, the r.m.s. difference between these two values is $\sim 27^{\circ}$.

The percentage of polarization of the BLR is generally smaller than that measured in the continuum (Figure 5, right panel). The median ratio $P_{\text {cont }} / P_{\mathrm{BLR}}$ is 1.7 . Furthermore, as discussed above, the dilution from the host galaxy in the red, low luminosity QSOs (the empty symbols) can lead to an underestimate of $P_{\text {cont }}$, further increasing the difference between continuum and BLR polarization.

\subsection{The radio-loud QSO}

There are 9 radio-loud QSO in our sample. Their radio images from the FIRST are presented in Figure 6. They are all associated with extended radio sources, with the only exception being J140700, a young GHz-peaked spectrum source (de Vries et al. 1997) that, nonetheless, has one of the largest value radio-loudness $(R=580)$.

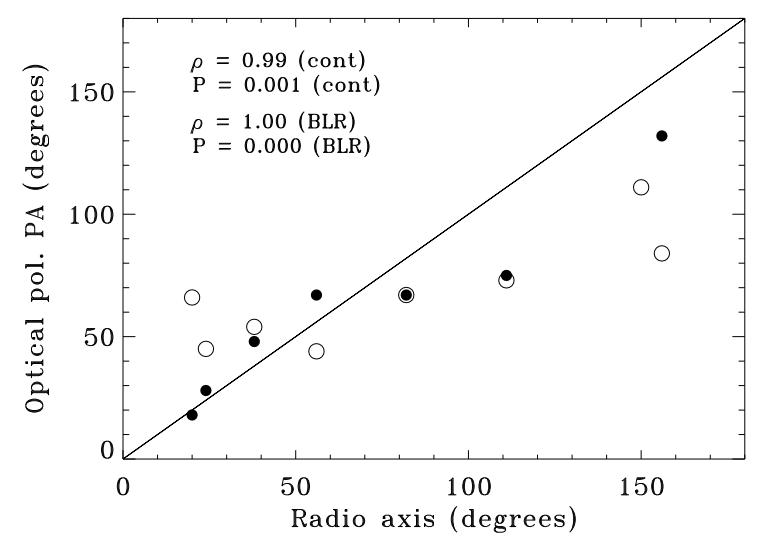

Figure 7. Comparison of the PA of the radio emission and the optical continuum polarization (filled dots) and of the BLR (empty dots) for the resolved radio loud QSO. The PA of the Radio and the optical continuum polarization differ by $18^{\circ}$ on average. The parallel position angles indicate the scattering is by an equatorial medium, in a plane perpendicular to the jet axis.

Figure 7 compares the radio axis and optical continuum polarization PA for the RLQs: their difference is always smaller than $\sim 35^{\circ}$, with a r.m.s. of $\sim 18^{\circ}$. The BLR polarization PA (including J110538 for which $\mathrm{PA}_{\text {cont }}$ cannot be measured) is also aligned with the radio axis, with a larger r.m.s. difference of $\sim 31^{\circ}$.

A further connection emerges when comparing the polarization and radio properties of the RLQs. A suggestive trend emerges between their radio core dominance, defined as the ratio between the nuclear core component in the FIRST images and the total NVSS flux (both at $1.4 \mathrm{GHz}$ ) and the continuum polarization (see Figure 8), which decreases for larger values of core dominance. The Spearman rank test indicates a moderate negative correlation between these two quantities with $\rho=-0.62$ and a chance probability of 0.10 . The low optical polarization of the high core dominance objects suggest the polarization is induced by scattering from 


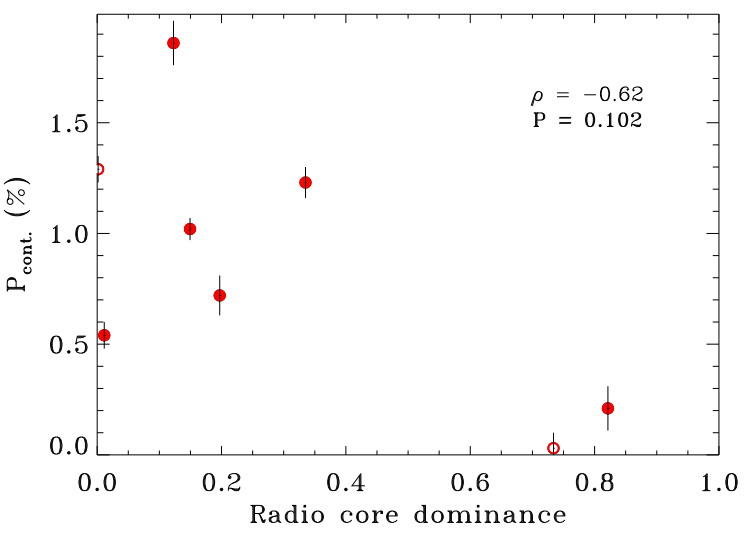

[h]

Figure 8. Comparison of the radio core dominance (a proxy for the jets orientation) and the optical continuum polarization for the radio loud QSO. A moderate negative correlation is present $(\rho=-0.62, \mathrm{P}=0.10)$. The empty dots mark the two red low luminosity RLQs. J140700, which is a young GHz-peaked spectrum source (de Vries et al. 1997), is omitted from this plot, as its compactness is physical and not a projection effect. The low optical polarization of the high core dominance objects suggest the polarization is induced by scattering from a medium with an axial symmetry, presumably due to cancellation in a face-on geometry.

a medium with an axial symmetry, presumably due to cancellation in a face-on geometry.

\section{SPECTRALLY RESOLVED POLARIZATION MEASUREMENTS}

Due to the general low level of polarization of the sources, spectrally resolved polarization measurements must be obtained by decreasing the resolution of the data. The spectra were rebinned in order to obtain, where possible, statistically significant measurements. The bin sizes used are smaller across the BLR and they increase far from the spectral regions of interest. Initially, the bin size was set to $10 \AA$ (rest frame, corresponding to $\sim 450 \mathrm{~km} \mathrm{~s}^{-1}$ ) the original instrumental resolution of the spectra. Five narrow bins across the core of the BLR enable us also to separate the possible polarization contribution of the $\mathrm{H} \alpha$ and $[\mathrm{N} \mathrm{II}]$ narrow lines. At larger spectral distances from the BLR core, the $10 \AA$ grid is maintained when the continuum subtracted polarized flux has a significance $>3 \sigma$. When this requirement is not met, the bin size is increased until 1) the required significance is reached or 2) when the bin width reaches half its velocity difference from the BLR core: i.e., a bin whose center is located $100 \AA$ from the BLR core cannot be broader than $50 \AA$. This strategy maintains a fixed minimum relative velocity resolution across the binned spectra. The procedure continues at larger velocity offsets on both sides of the spectrum independently.

In Figure 9 we present one example of the results obtained, i.e., the Stokes parameters, the polarized flux $I_{P}$, the degree of polarization $P$, and the polarization angle over a spectral region extending $\pm 600 \AA$ (rest frame, $\sim 27,000 \mathrm{~km} \mathrm{~s}^{-1}$ ) on each side of the $\mathrm{H} \alpha$ line center. The continuum subtracted spectra are shown in the right panels. Here we use the continuum PA as reference for the angles. The results for all sources are shown in the Supplementary Material. In Figure 10 we show a sub-set of the spectra obtained, comparing the polarized flux after continuum subtraction with the total flux for all sources.

A common feature in the spectra is a sign change of $Q$ and $U$ across the broad $\mathrm{H} \alpha$ line profile, corresponding to a rotation of the PA. In order to estimate the PA swing across the BLR we integrated the Stokes parameters on the red and blue sides of the broad $\mathrm{H} \alpha$ line. We experimented with various integration windows. The results reported refer to the case in which we split the BLR into a blue and a red region, each of them with a width equal to the $\mathrm{H} \alpha$ FWHM. This measurement is possible for 13 out of 25 sources in which the polarization is defined on both regions with a significance larger than $2 \sigma$.

Note that in two sources (J031027 and J105151) the BLR polarization is very low and we cannot measure the integrated BLR PA. Nonetheless, we were able to estimate the PA swing. In these sources the very large differences in PA causes polarization cancellation when integrating the whole BLR, but the individual wings are significantly polarized.

Figure 11 compares the amplitude of the PA swings, $\Delta P A_{\mathrm{BLR}}$, and the continuum polarization. The largest swings are seen in the least polarized sources, while sources with $P_{\text {cont }} \gtrsim 1 \%$ have swings smaller than $\lesssim 30^{\circ}$. The Spearman rank test indicates a significant negative correlation between these two quantities: for the 13 QSOs for which the PA BLR swing can be measured, we obtain $\rho=-0.63$ and a chance probability of $\mathrm{P}=0.02$. If the red low-luminosity QSOs are excluded, the correlation values are $\rho=-0.76$ and $\mathrm{P}=0.011$ for 10 objects. $\Delta P A_{\mathrm{BLR}}$ is not significantly correlated with the $\mathrm{H} \alpha$ width $(\rho=-0.28, \mathrm{P}=0.33)$, but, nonetheless, the largest swings are seen in the sources with FWHM $\lesssim 6000 \mathrm{~km} \mathrm{~s}^{-1}$.

The PA swings across the BLR suggest that the lower integrated BLR polarization with respect to the continuum discussed in Sect. 4.2 might be due to cancellation, resulting from the presence of polarization vectors oriented at different angles. To test this idea we introduce a different estimate of the BLR polarization: instead of integrating the Q and U Stokes parameters over the BLR spectral region, we measured the "average" percentage of BLR polarization from the $P$ values obtained from the rebinned spectra. These measurements are reported in Table 3, where we also give in parenthesis the observed rms of $P$ across the BLR. While these values are affected by the polarization positive bias, they are less influenced by the PA changes. We then tested the importance of cancellation by comparing the ratio between integrated and averaged BLR polarization and the PA swing across the BLR (see Fig. 12). Apparently, the sources with the largest angle swings are those where the BLR polarization is more reduced respect to the averaged value. Indeed, a strong significant negative $(\rho=-0.64, \mathrm{P}=0.018$ for 13 objects) trend is present.

\subsection{A comparison between the BLR width in total flux and polarized light}

We now estimate the width of the broad $\mathrm{H} \alpha$ line in total flux and in polarized light. The methods commonly used to measure the BLR width are the estimates of its FWHM or dispersion $\sigma_{\text {BLR }}$. Both have disadvantages, in particular when dealing with noisy spectra. For example, the measure of $\sigma_{\mathrm{BLR}}$ gives a strong weighting to the values of pixels in the wings of the lines, making it an unreliable estimator of line-width in low $\mathrm{S} / \mathrm{N}$ spectra. In the case of spectropolarimetric measurements, we are dealing not only with low S/N data, but they are also affected by the polarization bias (Wardle \& Kronberg 1974; Vaillancourt 2006): the polarization is 

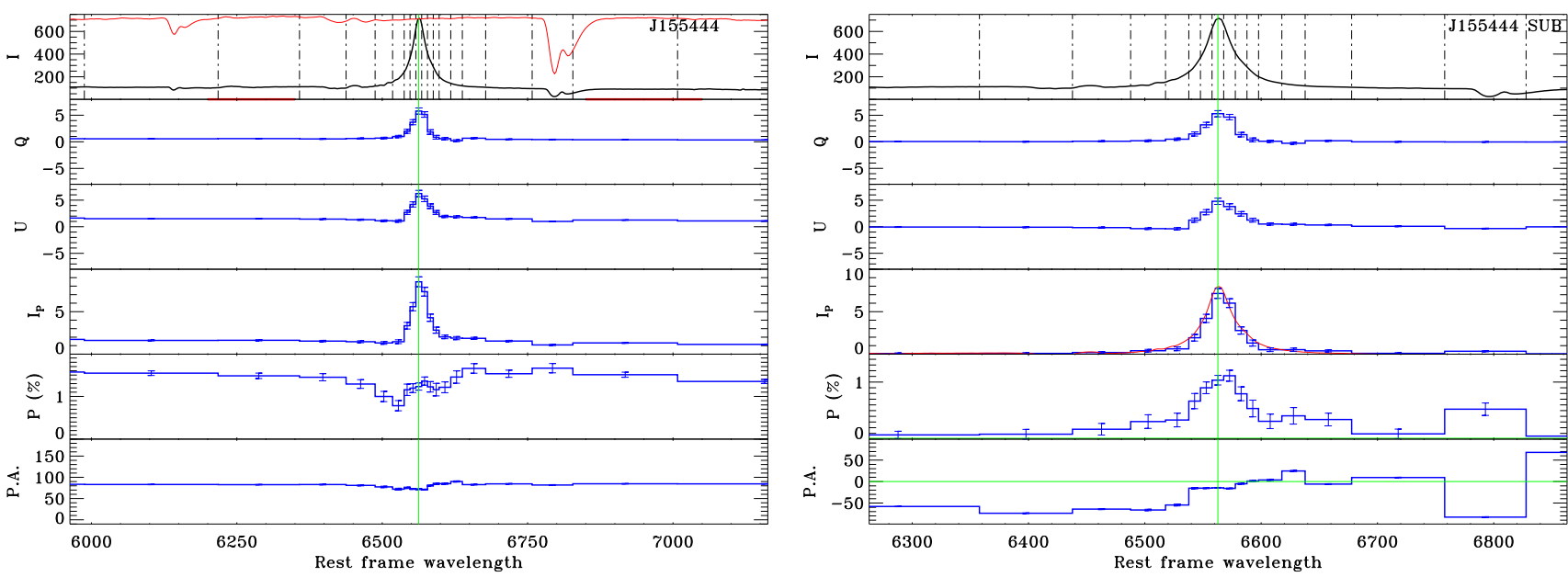

Figure 9. Example of the spectropolarimetric parameters obtained for each source of the sample, here we show the results for J155444. Left: top three panels: I, Q, and U Stokes parameters shown over a spectral region $1200 \AA$ wide. I is shown at full spectral resolution, the vertical dashed lines mark the boundaries of the regions used for the rebinning. Wavelengths are rest frame in $\AA$, fluxes are in arbitrary units. The green vertical line marks the location of the H $\alpha$ line. The red curve in the top panel represents the ratio between the SDSS and the corrected VLT spectra in which can be located the telluric absorption bands. The two horizontal red lines locate the spectral regions used to estimate the continuum polarization. Bottom three panels: polarized flux $I_{P}$, percentage of polarization $P$, and polarization position angle PA. Right: same as the left panels, but after subtraction of the continuum polarization and with a reduced spectral region (600 $\AA$ wide). The total flux is plotted in red over the polarized flux. The PA is now measured with respect to the continuum PA.

the length of a pseudo-vector, i.e., a definite positive quantity. As a consequence, the polarization measurements follow a Rice (and not a Gaussian) distribution. A direct measurement of the width of a line in polarized light, both the FWHM or $\sigma_{\mathrm{BLR}}$, is generally unreliable.

Following Whittle (1985), we used instead the inter-percentile values (IPV) to characterize line widths (see also Fine et al. 2008). The dependence of IPV widths on the cumulative flux distribution rather than on the flux density at a given point makes the IPV measurements more robust. IPV widths, $W$, can then be directly compared to the values derived from any BLR model or a different width measurement. For example, for a Gaussian profile, FWHM = $1.75 \times W$.

For each of the 25 QSO we built the cumulative distribution of the total flux $I$ and of the polarized flux $I_{P}$ and measured the wavelengths of the $25^{\text {th }}, 50^{\text {th }}$, and $75^{\text {th }}$ percentiles, $\lambda_{25}, \lambda_{50}$ and $\lambda_{75}$. In order to reduce the effects of noise and of the polarization bias it is necessary to consider the rebinned spectra. As a consequence the cumulative distribution is coarsely defined. The percentile values are estimated by performing linear interpolations between the adjacent measurements. The width between $\lambda_{25}$ and $\lambda_{75}$ is $W$ while $\lambda_{50}$ corresponds to the line centroid. For the sake of simplicity we refer to the IPV width measurements in direct and polarized light as $W(I)$ and $W\left(I_{P}\right)$, respectively.

IPV widths are also affected by the choice of the integration region which is not known a priori. For each source we considered, as first guess, a wavelength range of three times the FWHM of the broad $\mathrm{H} \alpha$ line in total flux and measured the line width in polarized light. In a second iteration, we used as wavelength range three times the value of $W\left(I_{P}\right)$ estimated in the first iteration. We proceed until convergence, which required at most three iterations.

We estimated the error on $W\left(I_{P}\right)$ with a Monte Carlo simulation. While the polarization measurements follow a Rice distribution, the individual Stokes parameters obey Gaussian statistics. We perturbed $Q$ and $U$ independently (the errors in $I$ are negligible) in each pixel of the original spectra by adding to the data a gaussian noise with amplitude given by the error at each given wavelength, producing 100 different realizations. The resulting spectra are then treated with the same strategy described in Sect. 2 and 3, including the adaptive rebinning and the subtraction of the continuum polarization. The $W\left(I_{P}\right)$ width in polarized light is measured for each realization and from their distributions we estimated the $1 \sigma$ error. The relative errors are between $\sim 10 \%$ and $\sim 25 \%$.

The resulting values of $W(I)$ and $W\left(I_{P}\right)$ are tabulated in Table 3 where we also give the shift between the centroid of the line in total and in polarized light. In Figure 13 we compare the broad $\mathrm{H} \alpha$ FWHM to the separation between the $25^{\text {th }}$ and $75^{\text {th }}$ percentiles, W(I). Overall, they show a ratio of 1.75 , but the objects with FWHM $\lesssim 4,000 \mathrm{~km} \mathrm{~s}^{-1}$ show smaller values, with FWHM/W(I) between 1.0 and 1.3. This effect is due to the well known connection between the line width and its profile, where the broad-line objects have more flat-topped profiles, while the narrowline objects have more extended wings (e.g., Collin et al. 2006).

In Figure 14, left panel, we compare the width measured in polarized light $W\left(I_{P}\right)$ and total flux $W(I)$ : the line width in polarized light is, in all but four QSOs, significantly broader than in total flux with a broadening reaching a factor $\sim 2.5$. In many cases, a large line broadening is associated with a double-humped profile in polarized light. The median value of the broadening is $\sim 1.5$.

In the right panel we instead compare the shift between the BLR centroid in total and polarized light with the total flux width. Most QSOs do not show a statistically significant shift, with values of shift smaller than $\sim 1000 \mathrm{~km} \mathrm{~s}^{-1}$ and no relation with $W(I)$. However, there are three sources, among those with the largest line widths $\left(W(I) \sim 5000 \mathrm{~km} \mathrm{~s}^{-1}\right)$, showing large shifts. In particular, a highly significant redshift of $2,050 \pm 350 \mathrm{~km} \mathrm{~s}^{-1}$ is measured in J140336. These three QSOs are all radio-loud and do not show a significant line broadening, i.e., $W\left(I_{P}\right) / W(I) \sim 1$.

In Figure 15 we compare the broadening, i.e. the ratio between $W\left(I_{P}\right)$ and $W(I)$, with the percentage of polarization of the continuum and the PA swing across the BLR. In both cases the two quantities are strongly correlated: the sources with the largest broadening are the least polarized $(\rho=-0.63$ and $\mathrm{P}=0.001$ for 25 


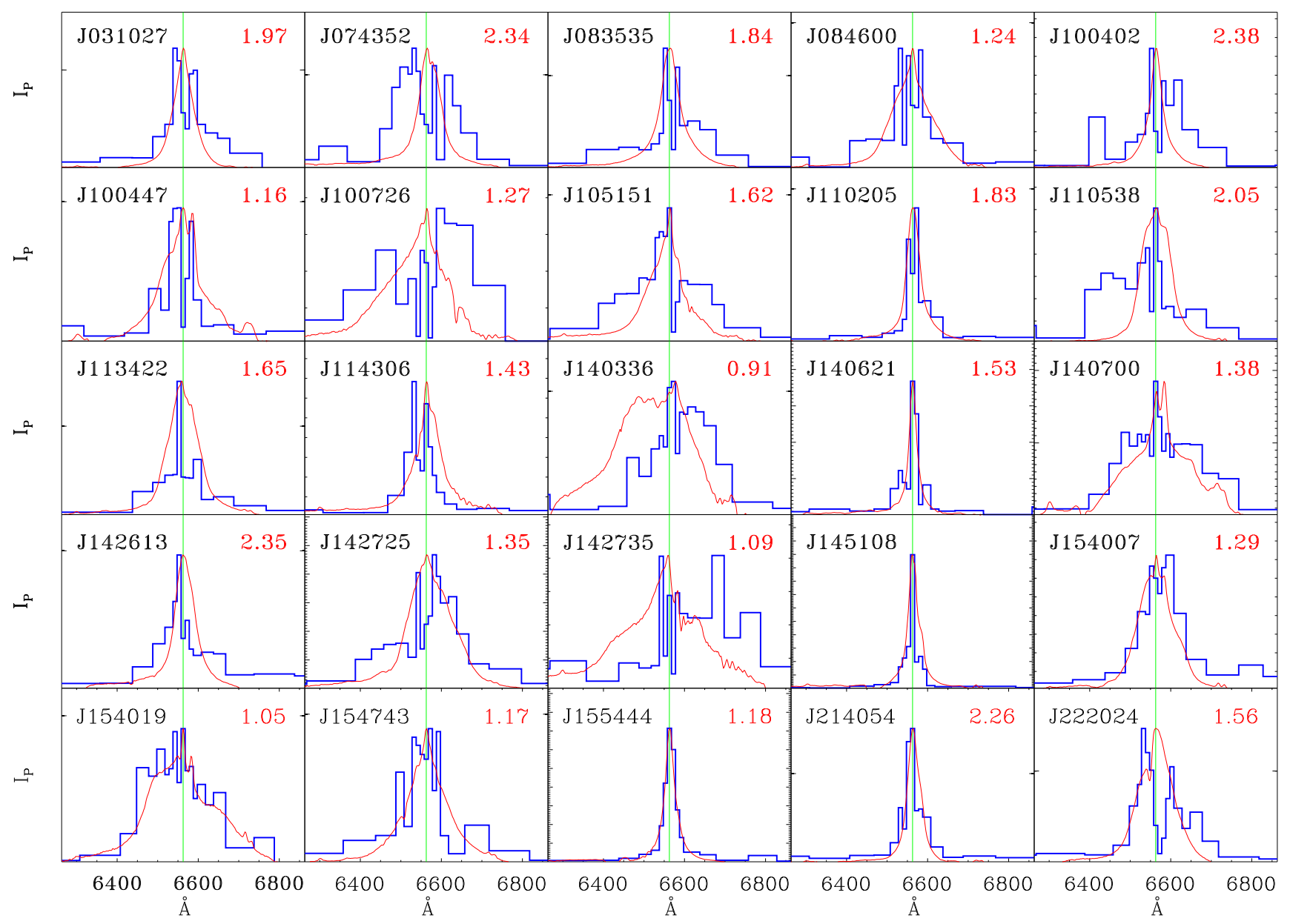

Figure 10. Summary of the spectropolarimetric results for the 25 QSOs. The red curve shows the total flux, the blue histogram the polarized flux after continuum subtraction. The green vertical line marks the location of the narrow $\mathrm{H} \alpha$ line. The red number on the top right of each panel shows the ratio between the width in polarized and in total flux $\left(W\left(I_{P}\right)\right.$ and $W(I)$, respectively; see below for details). Note that this ratio is generally above unity. This reflects the higher velocity dispersion from the equatorial vantage point of the scattering medium, compared to the direct view. The broadest line objects (J100447, J140336, J142735, J154019) show a ratio close to unity, which suggest the direct view is close-to equatorial. The narrower line objects generally show a large ratio (e.g. J100402, J214054), indicating a close-to face-on view, but some have a low ratio (e.g. J155444), indicating a high inclination view. The scattered line is significantly shifted to the red in some objects (J140336, and possibly J100726 and J142735), which may indicate scattering from an outflow. The full spectropolarimetry results are presented in the Supplementary Material

sources) and with the largest PA swing across the BLR ( $\rho=0.81$ and $\mathrm{P}=0.0008$ for 13 sources).

A slightly different view of the same effect is obtained by looking at the dependence of the line broadening with the FWHM, taking into account also the continuum polarization (see Fig. 16). The very broad line objects have small broadening which indicates the objects are observed at a high inclination. These objects also have a high continuum $\% \mathrm{P}$, as expected for their high inclination. At low FWHM values, there is a large scatter in $W\left(I_{p}\right) / W(I)$, which indicates it is a mix of objects observed over a wide range of inclinations. The objects with highest $W\left(I_{p}\right) / W(I)$ at low FWHM are expected to be closer to face-on, as indeed indicated by their low $\% \mathrm{P}$.

\section{THE ORIGIN OF THE POLARIZATION IN QSOS}

We here summarize the main results obtained in the previous sections.

- The continuum \%P and PA appears to remain steady over timescales of years, as indicated by a comparison to the values measured $\sim 30-40$ years ago for seven sources from our sample.

- The median continuum polarization is $0.59 \%$. The QSOs with the lowest luminosities are redder and less polarized $(\bar{P}=$ $0.39 \%)$ compared the more luminous sources $(\bar{P}=0.72 \%)$. The difference is most likely due to the significant contribution of the unpolarized host galaxy light, which reddens the observed continuum, and lowers its polarization.

- When integrated over the broad $\mathrm{H} \alpha$ profile, the BLR PA is closely aligned with the continuum PA. But, $P_{\text {cont }}$ is higher than the average $P_{\mathrm{BLR}}$ by a factor of $\sim 2$.

- RLQs show a close alignment between their radio axis and both the continuum and BLR polarization.

- RLQs show an inverse relation between $P_{\text {cont }}$ and the radio core dominance, where $P_{\text {cont }}$ drops with increasing core dominance.

- A significant swing of the polarization PA vector is commonly observed across the BLR profile. The largest swings are seen at the lowest $P_{\text {cont }}$ and the lowest $\mathrm{H} \alpha$ FWHM. 

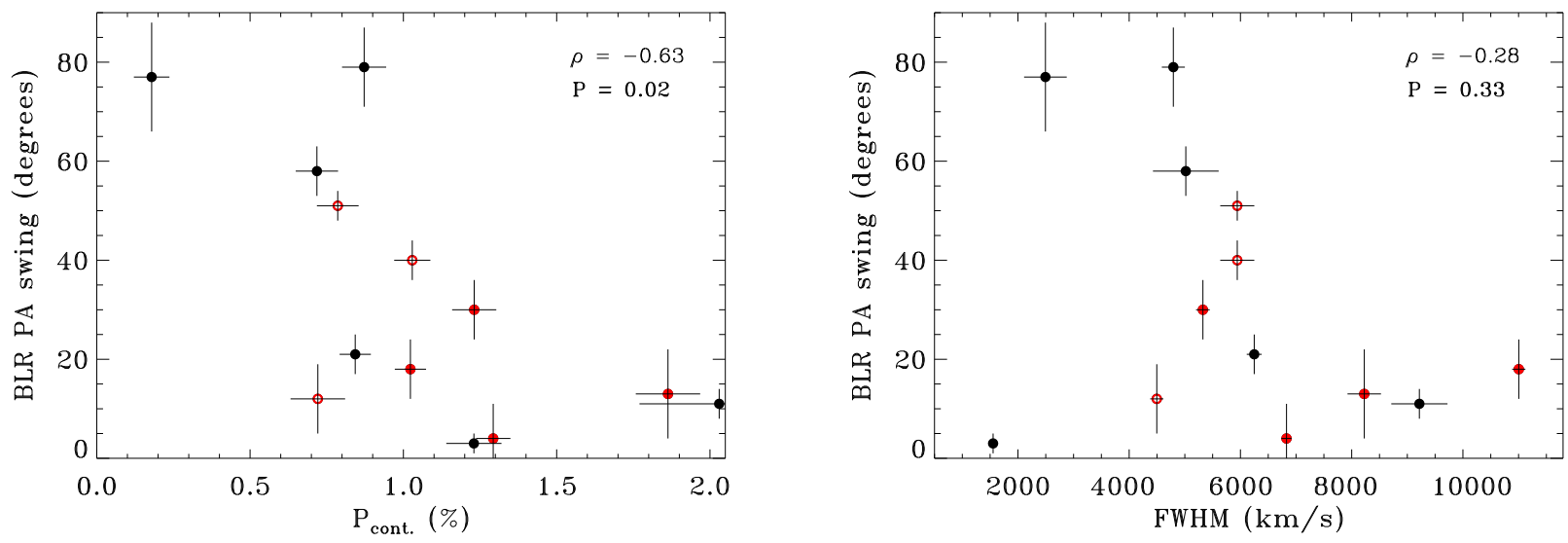

Figure 11. The dependence of the swing in the polarization PA between the red and the blue wing of $\mathrm{H} \alpha$ on: (left) the continuum polarization and (right) the $\mathrm{H} \alpha$ line width. There is a highly significant negative correlation between PA swing and continuum polarization, as expected for an equatorial scattering medium. The relation of the PA swing and line width is weaker, as the line width is affected by both the inclination and the black hole mass. The largest swings are seen in the sources with FWHM $\lesssim 6000 \mathrm{~km} \mathrm{~s}^{-1}$, which suggests they are all high inclination objects (see also Figure 16). The low luminosity red QSOs, with $\alpha_{\text {opt }}>-1$ and $\log L_{5100}<\tilde{44.5}$, are represented by empty circles (see Figure 4 ). The red symbols are the RLQs.

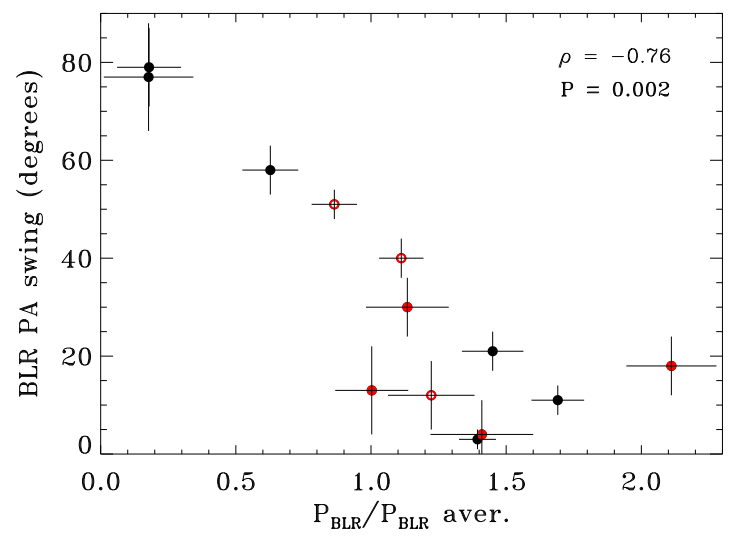

Figure 12. The ratio between the $\mathrm{H} \alpha$ polarization, which is measured from the integrated line flux, and the averaged wavelength dependent $\mathrm{H} \alpha$ polarization, versus the PA swing. The tight correlation indicates that the integrated $\mathrm{H} \alpha$ polarization is strongly affected by the PA swing cancellation (i.e., summing polarization vectors oriented at different angles), which explains some of the reduced $\mathrm{H} \alpha$ polarization compared to the continuum polarization.

- The broad $\mathrm{H} \alpha$ line width in polarized light is generally broader than in direct-light, by a median factor of $\sim 1.5$. The largest BLR broadening in polarized light is observed in the objects with the lowest $\mathrm{H} \alpha \mathrm{FWHM}$, the lowest $P_{\text {cont }}$, and the largest PA swing.

- The polarized broad $\mathrm{H} \alpha$ line profile of $\mathrm{J} 140336$ is redshifted by $\sim 2,000 \mathrm{~km} \mathrm{~s}^{-1}$ with respect to the total flux profile, but the line width is essentially unchanged, in the highest $\mathrm{H} \alpha$ FWHM object. A similar effect may be present in two other high $\mathrm{H} \alpha$ FWHM objects.

- In most cases, however, the shift between the broad $\mathrm{H} \alpha$ line in total and polarized light is not statistically significant, with values of shift smaller than $\sim 1000 \mathrm{~km} \mathrm{~s}^{-1}$ and no relation with $W(I)$.

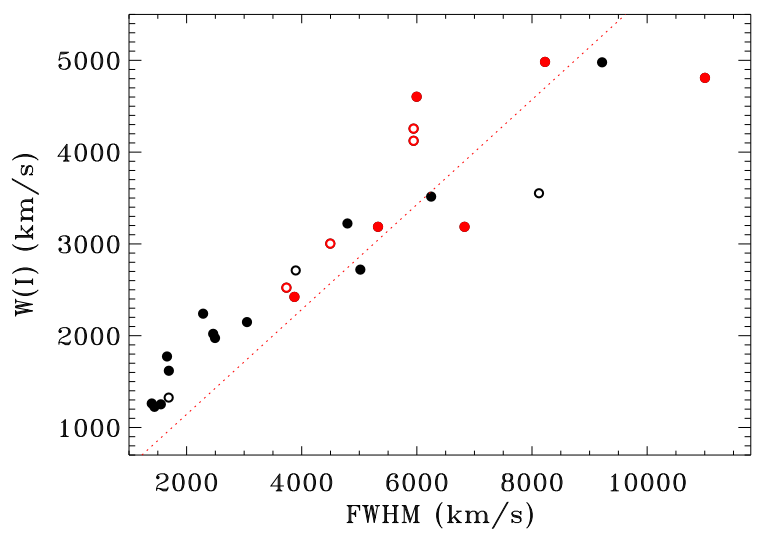

Figure 13. Comparison of the broad $\mathrm{H} \alpha \mathrm{FWHM}$ and $W(I)$ - the IPV width, which is the separation between the $25^{\text {th }}$ and $75^{\text {th }}$ percentiles of the integrated line flux. The two parameters follow roughly a ratio of 1.75 (dotted line). The ratio is closer to unity at the lowest FWHM objects, due to the more prominent line wings in these sources. Thus, although $W(I)$ provides a good measure of the line width, it can deviate systematically from the FWHM in very narrow or very broad line objects.

- The 16 RQQs and 9 RLQs follow in a similar manner the various relations described above.

- The interstellar polarization has, in general, a negligible effect on the polarization properties of the QSOs we observed.

There is a host of mechanisms which can induce line and continuum polarization in AGN. The continuum polarization may be produced by synchrotron emission. The thermal disk emission may be polarized by electron scattering within the disk atmosphere (e.g., Laor et al. 1990; Agol \& Blaes 1996). The BLR emission lines may be resonantly, Rayleigh, or Ramann scattered and thus polarized by ambient gas clouds (Lee \& Blandford 1997; Lee \& Yun 1998; Korista \& Ferland 1998; Chang et al. 2015). Transmission of the quasar emission through aligned dust grains along our line-of-sight can polarize all the quasar emission (Serkowski et al. 1975). In ad- 

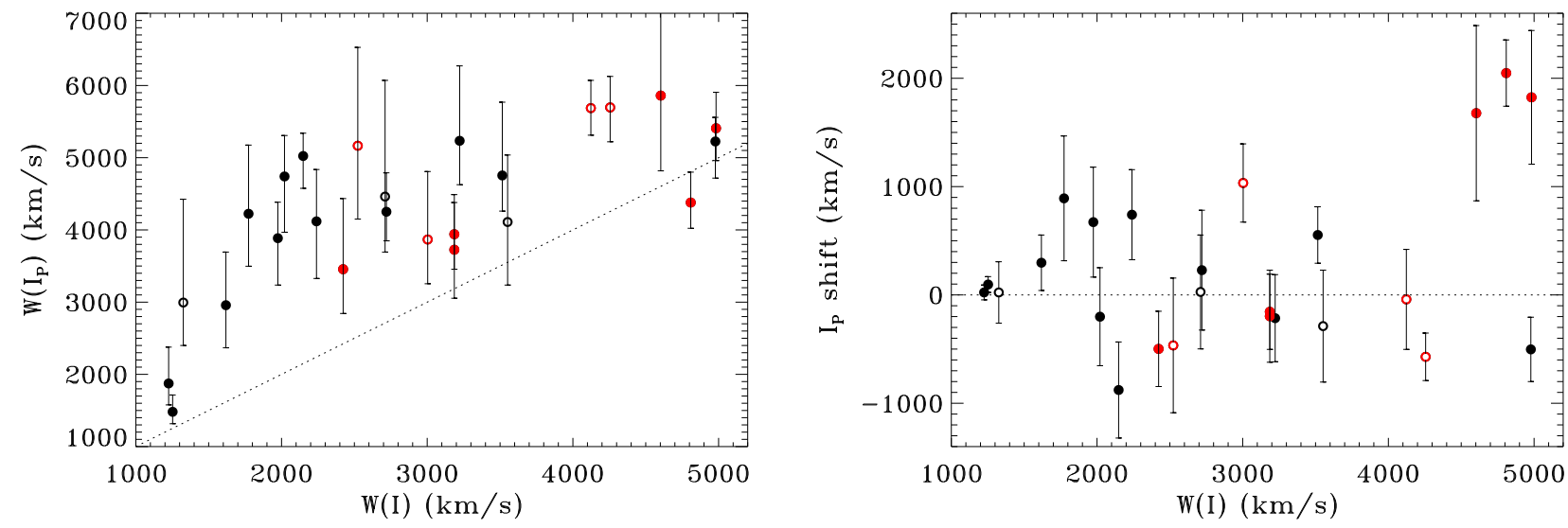

Figure 14. Left: comparison of the IPV widths measured in total flux and polarization, $W(I)$ and $W\left(I_{P}\right)$, respectively. There is a general broadening of the BLR in polarized light with respect to what is seen in total flux. Right: velocity shift, in $\mathrm{km} \mathrm{s}^{-1}$, between the BLR centroid in $I_{p}$ with respect to the BLR in total flux. Polarized line shifts are generally small $\left(\lesssim 1000 \mathrm{~km} \mathrm{~s}^{-1}\right)$ and rarely significantly different from a null value, but there is a group of three RLQs with a more significant shift of $\sim 2000 \mathrm{~km} \mathrm{~s}^{-1}$.
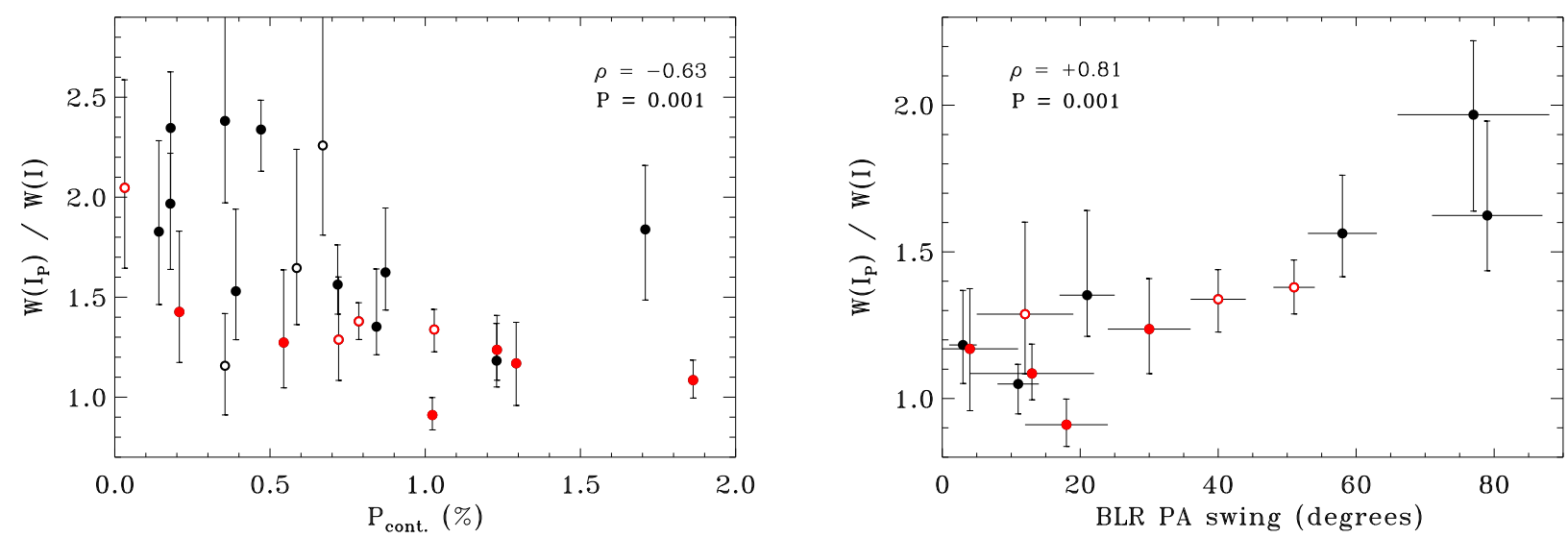

Figure 15. The polarization broadening of the BLR, i.e., the ratio between the line width in polarized and total flux versus: (left) the percentage of polarization of the continuum, and (right) the PA swing across the BLR. The sources with the largest broadening are the least polarized and with the largest PA swing across the BLR. Both trends are expected for equatorial scattering observed at a range of inclinations. With decreasing inclination, $W(I)$ becomes smaller, while $W\left(I_{p}\right)$ is unchanged, so $W\left(I_{p}\right) / W(I)$ increases, the PA swing is also expected to rise, while the polarization strength decreases.

dition, continuum and BLR polarization can be induced through electrons or dust grain scattering, as commonly observed in type 2 AGN, the phenomena which underlies the AGN unification scheme (Antonucci 1993).

The similarity of the PA of the continuum and the BLR suggests that the same mechanism is responsible for the polarization of both components. This excludes the separate line and continuum polarization mechanisms, as mentioned above. Despite the similarity in PA, the mean amplitude of $P_{\text {cont }}$ is different from the amplitude of $P_{\mathrm{BLR}}$, which excludes dust transmission, which would produce the same polarization of all the transmitted radiation.

The only remaining viable mechanism is scattering, either by free electrons or dust. Scattering induced polarization depends on the geometry of the scattering configuration. Since AGN are characterized by axial symmetry, the geometry can be ideally separated into two situations, polar and equatorial scattering (spherically symmetric scattering produces no polarization). The align- ment of the polarization PA and the radio axis PA indicates equatorial scattering. Since the RLQs and RQQs follow the various relations found here in a similar manner, the polarization mechanism for both types of AGN is likely similar. We therefore conclude that equatorial scattering applies for both types of quasars.

Furthermore, scattering can be divided into a near-field and a far-field regime. The near-field regime occurs when the emission region and the scattering regions overlap or are next to each other. The detection of a swing of the PA across the BLR in most QSO indicates that we are generally observing near-field equatorial scattering.

Further support for the near field equatorial scattering, is the additional observation that the average $P_{\mathrm{BLR}} \sim 0.5 P_{\text {cont }}$, which is naturally interpreted as a geometrical cancellation effect. Different emitting regions in the BLR are scattered at different angles from a given region in the extended scattering region (see Smith et al. 2005), which lowers the polarization of the integrated flux scat- 


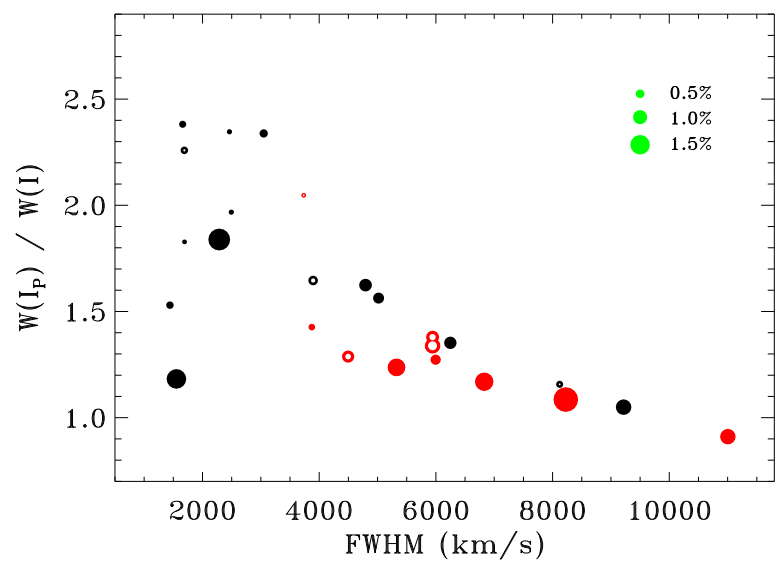

Figure 16. The polarization broadening of the BLR, i.e. $W\left(I_{p}\right) / W(I)$, as a function of the $\mathrm{H} \alpha$ FWHM. Symbols sizes are proportional to the continuum polarization. The very broad line objects (FWHM $>6500 \mathrm{~km} \mathrm{~s}^{-1}$ ) have small broadening $(<1.2)$ which indicates the objects are observed at a high inclination, close to the inclination over which the equatorial scattering medium extends. These objects also have a high continuum \%P, as expected for their high inclination (the one deviant object with a low $\% \mathrm{P}$ is host diluted). At low FWHM values, there is a large scatter in $W\left(I_{p}\right) / W(I)$, which indicates it is a mix of objects observed over a wide range of inclinations. The objects with highest $W\left(I_{p}\right) / W(I)$ at low FWHM are expected to be closer to face-on, as indeed indicated by their low \%P. The highest $\%$ P objects at low FWHM have lower $W\left(I_{p}\right) / W(I)$, which both indicate a higher inclination.

tered. In contrast, the continuum source is far smaller than the scattering region, and all parts of the continuum emission region are scattered by the same set of scattering angles in the scattering medium, which is less affected by the geometrical cancellation effect.

A key additional observable, in the case of near field scattering, is the PA swing across the BLR, $\triangle \mathrm{PA}_{\mathrm{BLR}}$. The models indicate that this is related to the orientation of the BLR with respect to our line-of-sight: at smaller inclinations we expect larger $\triangle P A_{\mathrm{BLR}}$ and smaller $P_{\mathrm{cont}}$. This relation is indeed found here, where Fig. 11 demonstrates a highly significant negative correlation between $P_{\text {cont }}$ and $\triangle \mathrm{PA}_{\mathrm{BLR}}$. The specific velocity dependence of $\mathrm{PA}_{\mathrm{BLR}}$ is consistent with the predicted PA velocity dependence for a rotating disk emitter, scattered by an outer rotating disk medium (Smith et al. 2005; Savić et al. 2018; Lira et al. 2020). The maximal observed PA swing of $\sim 80^{\circ}$ found here, which is expected for a close-to face-on view, suggests the scattering medium radius is $\sim 1.5 \times$ the size of the emitting region (see Fig. 6 in Smith et al. 2005), so the scattering medium may be effectively considered as the outer BLR.

Polarization angle swings in the $\mathrm{H} \alpha$ profile might also cause cancellation because polarization vectors of different orientation are added up when integrating over a large velocity range. Indeed we found that sources with the largest ratio between the average and the integrated $P_{\mathrm{BLR}}$ are those with the largest PA swings: the reduced level of polarization integrated over the whole BLR is indeed due to this effect.

The importance of orientation on the polarization properties of QSOs is also suggested by the connection between $\triangle P_{B L R}$ and the BLR width. Larger $\triangle \mathrm{PA} \mathrm{BLR}_{\mathrm{B}}$ are generally found in QSOs with a smaller FWHM, indicating that orientation affects the observed line width. However, this is not a one-to-one correspondence, as there are sources with small $\triangle \mathrm{PA}_{\mathrm{BLR}}$ associated with a smaller FWHM. This is expected because the BLR width distribution is produced by the folding of intrinsic widths with orientational effects.

Further support for the idea of equatorial scattering and, at the same time, another signature of the importance of orientation is found when considering the broadening of the BLR in polarized flux compared to total flux. The polarized broad lines result from scattering by material distributed mainly in the AGN equatorial plane. From this vantage point, the rotational component of the velocity is maximized and it is then higher than along our lineof-sight. The broadening reaches values as large as $\sim 2.5$, with a median value of $\sim 1.5$. A significant negative trend links the broadening with the $\mathrm{H} \alpha \mathrm{FWHM}$, and with the continuum polarization, and a strong positive trend is seen between the broadening and PA swing across the BLR. The three effects are all expected for equatorial scattering: at smaller inclination the polarization and the typical FWHM decreases, while the BLR PA swing and the line width ratio in total to polarized light, both increase.

The median broadening expected for a random orientation of the QSOs is equal to $1 / \sin \left(60^{\circ}\right) \sim 1.15$, smaller than the observed value of $\sim 1.5$ that corresponds instead to a median viewing angle of $\sim 42^{\circ}$. This effect is likely due to the presence of circumnuclear selective obscuration, as predicted by the unified model of active nuclei. Another indication of the role of orientation, already discussed in Sect. 4.3, is the negative correlation between degree of polarization and radio core dominance in the RLQs: the former increases at larger viewing angles while the latter decreases.

Thus, there is a single scenario which explains the polarization properties of this representative sample of QSO. The polarization of both the continuum and the BLR are produced by an equatorial scattering medium which resides close, or possibly at, the BLR.

While the polarization properties of most of the QSO in this sample are well described by near-field equatorial disk scattering, there are some deviants. For example, in J142613 the continuum PA and the integrated $\mathrm{H} \alpha$ flux PA are orthogonal. The very low $\% \mathrm{P}(<0.2 \%)$ of both the continuum and the BLR, and the non physical erratic structure in the wavelength dependence of the BLR PA (see Supplementary Material), suggest possible systematic effects. However, some variations in the equatorial scattering scheme are also possible.

Another variation is observed in J140336. Its polarized BLR is redshifted by $\sim 2,050 \pm 310 \mathrm{~km} \mathrm{~s}^{-1}$ with respect to the total flux BLR. The BLR profile, leaving aside its redshift, is quite similar in both polarized and direct-light and, in particular it does not show a significant broadening $\left(W\left(I_{P}\right) / W(I)=0.91 \pm 0.07\right)$. There are two further sources with apparently similar properties, but with poorer data quality: J142735 where the polarized BLR is redshifted by $1,820 \pm 620 \mathrm{~km} \mathrm{~s}^{-1}$, and $\mathrm{J} 100726$ where the redshift is $1,680 \pm 810 \mathrm{~km} \mathrm{~s}^{-1}$. Their line broadenings are $1.09 \pm 0.10$ and $1.27 \pm 0.29$, respectively. These three sources have several characteristics in common: they are all radio-loud QSO and are the sources with the broadest BLR in this group, with a FWHM between 6,000 and 11,000 $\mathrm{km} \mathrm{s}^{-1}$, among the QSO with the highest luminosity, and are likely to be observed closer-to edge-on (high $\% \mathrm{P}$ and small core dominance). 


\section{DISCUSSION}

\subsection{Comparison with earlier studies}

Some of the relations found here were already noted in earlier studies. Specifically, the typical optical continuum polarization amplitude of QSOs, which is 0-2\% (Stockman et al. 1984; Berriman et al. 1990). The parallel alignment of the radio axis PA and the polarization PA in RL quasars (Stockman et al. 1979; Antonucci 1983; Berriman et al. 1990).

In contrast with our conclusion that the continuum and BLR polarization are produced by a single scattering medium, Kishimoto et al. (2004) suggest that the optical polarization in quasars is produced by electron scattering within the accretion disk atmosphere. The conclusion is based on the detection of a drop in the polarization amplitude below $4000 \AA$. This drop is interpreted as the Balmer edge opacity jump which may make the absorption opacity larger than the scattering opacity, and thus reduce the polarization induced by scattering (see a similar effect predicted at the Lyman edge, Laor et al. 1990). However, this interpretation is inconsistent with the PA of a disk atmosphere scattering, which is predicted to be perpendicular to the radio axis, rather than parallel, as observed. Furthermore, scattering from dust is expected to induce a drop in the polarization amplitude in the UV due to the wavelength dependence of the scattering cross section (Zubko \& Laor 2000). The exact wavelength dependence of the polarization amplitude depends on the dielectric function, i.e. the grain composition, and to some extent also on the grain size distribution.

A polarization PA swing across the $\mathrm{H} \alpha$ line in a fraction of type 1 AGN was noted in various studies (Goodrich \& Miller 1994; Corbett et al. 2000; Smith et al. 2002, 2004; Afanasiev et al. 2019). A PA swing is also observed in the rest frame UV broad lines (Alexandroff et al. 2018). As noted above, the polarization PA swing is consistent with near field equatorial scattering (Smith et al. 2005; Savić et al. 2018; Lira et al. 2020).

A redshifted polarized line profile, suggesting scattering from a fast nuclear outflow, was also found in Robinson et al. (1999) and Young et al. (2007) from spectropolarimetric observations in two QSOs, 4C 74.26 and PG 1700+518, as found here for the redshifted polarized line objects.

Despite the similarity to earlier studies, a major difference is that earlier high $\mathrm{S} / \mathrm{N}$ spectropolarimetric studies (e.g., Goodrich \& Miller 1994) tend to find a complex situation with a variety of polarization properties, suggesting a mix of polarization mechanisms. This is in contrast with the more coherent picture derived here. This likely results from the nature of the earlier samples, which include a greater variety of objects, including significantly host dominated AGN, reddened AGN, and very low $L / L_{\text {Edd }} \mathrm{AGN}$. The new aspect in this study is that it is based on a uniformly selected and complete sample of quasars, which extends evenly over a wide range of luminosity and line width, and is thus likely more representative of the more common quasars.

\subsection{Further support for near field equatorial scattering}

The various correlations found between the continuum $\% \mathrm{P}$, the BLR PA swing, and the line width ratio $W\left(I_{p}\right) / W(I)$, provide further independent support for the equatorial scattering scenario. The continuum $\% \mathrm{P}$ is expected to increase with inclination, the PA swing is expected to decrease with increasing inclination (Smith et al. 2005), and the line width ratio $W\left(I_{p}\right) / W(I)$ is ex- pected to decrease with inclination. All expected relations are indeed observed.

The relations of the above three quantities with the BLR FWHM are generally weaker, showing a growing dispersion with decreasing FWHM. This trend is indeed expected, as the FWHM is affected by both the inclination and the absolute gas velocity at the BLR, which is set by both the size of the BLR and the black hole mass. The highest FWHM values are expected to come from a combination of both a high velocity BLR, and a high inclination view. Indeed these objects all show high \% P, low BLR PA swing, and $W\left(I_{p}\right) / W(I)$ close to unity, as expected. In contrast, the lower FWHM values includes both low velocity BLR observed at a high inclination, and higher velocity BLR observed at low inclination. Indeed, the low FWHM QSOs show a large range in the continuum \% P, BLR PA swing, and in $W\left(I_{p}\right) / W(I)$. However, as expected the low FWHM objects with a high $P_{\text {cont. }}$. show a smaller $W\left(I_{p}\right) / W(I)$, and the reverse, which indicates the scatter at low FWHM is an inclination effect.

The large swing inevitably leads to a drop in the integrated BLR flux polarization, compared to the mean value of the wavelength dependent polarization (Fig. 12). Thus, the BLR needs to be adequately spectrally sampled in order to resolve its true polarization level.

Another more minor piece of supporting evidence for equatorial scattering is that the large redshift offset of the polarized profile is observed in objects with a high inclination (high continuum $\%$ P, small BLR PA swing, $W\left(I_{p}\right) / W(I) \sim 1$ ), as expected for a closer-to edge-on view of an equatorial wind.

Additional support for near field equatorial scattering in type 1 AGN is provided by the nature of the type 2 AGN polarization, which is produced by polar scattering (Antonucci 1983; Antonucci \& Miller 1985). The absence of the equatorial scattering polarization signature in type $2 \mathrm{AGN}$ indicates this polarized light is obscured together with the continuum and BLR emission. Thus, the scattering region in type 1 AGN must be physically close to the BLR. In addition, only a small minority of type 1 AGN appear to be dominated by polar scattering (Smith et al. 2004), despite the fact that the polar scattering medium must be directly observed in type $1 \mathrm{AGN}$, together with the equatorial scattering region. This indicates that the equatorial scattered flux generally dominates the polar scattered flux. The polar scattering is consistent with far field scattering, while the equatorial scattering is near field. Thus, one naturally expects the covering factor and thus the scattered flux of the equatorial scattering medium to dominate.

Reverberation mapping of the polarized versus the direct-light continuum in NGC 4151 by Gaskell et al. (2012) reveals that the scattering medium has the size comparable to the BLR. Clearly, additional reverberation mappings of the continuum and of the BLR polarized fluxes can measure directly the size and the spatial distribution of the scattering medium in AGN (e.g., Rojas Lobos et al. 2020).

We note in passing that intensive spectropolarimetric studies of broad absorption line quasars (Cohen et al. 1995; Goodrich \& Miller 1994; Ogle et al. 1999; Schmidt \& Hines 1999), lead to the same picture of an equatorial scattering medium.

\subsection{Implications for black hole mass estimates}

The relations found in this study show that the continuum $\% \mathrm{P}$, the BLR PA swing, and the line width ratio $W\left(I_{p}\right) / W(I)$, can all be used as independent inclination indicators in AGN. We also find evidence that the BLR FWHM is indeed affected by both inclina- 
tion and the absolute gas velocity at the BLR, as expected for an equatorial BLR structure.

In principle one can use the three quantities, the continuum $\% \mathrm{P}$, the BLR PA swing, and the line width ratio $W\left(I_{p}\right) / W(I)$, to derive the inclination, and use that to correct the observed FWHM to the absolute gas velocity. This will allow to mitigate the inclination bias in the BLR based black hole mass estimate. However, the BLR is likely not a thin disk, as it needs to subtend over a significant solid angle ( $\sim 0.3$, see Baskin \& Laor 2018), which means it forms a torus structure. This structure inevitably implies a random velocity component, which is a fraction of the planar Keplerian velocity. Since this random component may be object dependent, it is not straightforward to derive the absolute gas velocity from the observed FWHM. The simplest and most straightforward approach is just to use the line width ratio $W\left(I_{p}\right) / W(I)$ as the FWHM correction factor, as this directly measures the increase in the velocity spread from our vantage point, to a close-to equatorial point of view.

A practical procedure is to first use broad band polarimetry at a number of wavelengths, in order to estimate the continuum $\% \mathrm{P}$ level. If say $\mathrm{P}<1 \%$ than the black hole mass correction factor $\left(W\left(I_{p}\right) / W(I)\right)^{2}$ may become comparable or larger than the black hole mass uncertainty (factor $\sim 3$ ). This can then justify investing the significant telescope time required to get a high quality spectropolarimetry of the object, in order to measure $W\left(I_{p}\right) / W(I)$ as accurately as possible.

\subsection{Further questions}

Equatorial scattering at, or just outside, the BLR is the major polarization mechanism in quasars. However, this is clearly just the first order level approximation.

The various relations found here often show significantly offset objects, which indicates second order effects must be present. For example, compact RLQ which are beamed, like 3C273, are observed very close-to face-on, but still show optical polarization of a few tenths of percent, which cannot be due to scattering, so there may be some synchrotron contribution to the polarization. Of the six quasars with $\mathrm{P}_{\text {cont }}>1 \%$ and $\mathrm{P}_{\mathrm{BLR}}<0.5 \%$, five are RL (Fig. 5 ). Is their continuum polarization boosted by contribution from a synchrotron component? If yes, this synchrotron source should not be beamed, as these objects are generally lobe dominated (Fig. 6 and 8).

Is the scattering produced by dust or by free electrons? The available wavelength baseline is too small to test the two options, and one clearly needs to extends this study to the UV. However, since the scattering occurs on the BLR scale, or just outside, dust can already survive in the gas. In dusty gas dust inevitably dominates the scattering, given its optical opacity which is likely a factor $\sim 100$ larger than the electron scattering opacity $\left(\sim 10^{-22}\right.$ vs. $6.56 \times 10^{-25} \mathrm{~cm}^{2}$ per $\mathrm{H}$ atom, e.g. Baskin \& Laor 2018).

Are there additional "fake" NLS1 objects, like PKS 2004447 (Baldi et al. 2016)? In PKS 2004-447 $W\left(I_{P}\right) / W(I) \sim 6$, whereas here the maximal ratio found is $\sim 2.4$. Clearly, objects like PKS 2004-447 are not common in type 1 AGN. Since the typical covering factor in AGN is $\sim 0.3$ (Baskin \& Laor 2018), these objects are inevitably obscured in a close-to edge-on view. Also the random velocity component at the BLR will be of the order of $\sim 1 / 2$ of the Keplerian speed, which again precludes a very high $W\left(I_{P}\right) / W(I)$. The high $W\left(I_{P}\right) / W(I)$ value observed in PKS 2004-447 requires a thin scattering medium, and also a thin disk BLR, which are not common. But, may be more common in
RLQ, as these can be found at relatively low $L / L_{\mathrm{Edd}}$, which may be required for these properties (Baskin \& Laor 2018).

Inclination is clearly a fundamental property which controls the observed properties of AGN. Polarization is sensitive to the geometry of the system, and can thus serve as a sensitive inclination indicator. Various other methods were suggested to derive the inclination of individual objects (e.g., Fischer et al. 2013; Marin 2014). AGN show a set of emission line and continuum correlation, which are generally termed the EV1 set of correlations (Boroson \& Green 1992). It is of great interest to establish whether and how the polarization properties change along the EV1 sequence. Answering this question can provide important hints on the physical mechanisms which produce the EV1 sequence. We plan to compare the indications on orientation provided by polarization with other suggested indicators in a forthcoming paper.

Finally, it will be interesting to explore the spectropolarimetric properties of higher redshift, $\mathrm{z} \sim 2-3$. The primary line used for such studies is C IV at $1549 \AA$, that, however, appears to provide a significantly less accurate, and possibly biased, estimate of $M_{\mathrm{BH}}$ (Baskin \& Laor 2005). In fact, the C IV line is known to be blueshifted with respect to the lower-ionization lines, and often shows strong asymmetries, suggesting that non-gravitational effects affect its profile. Furthermore, the line properties appear to be connected with the object's Eddington ratio. Spectropolarimetry of a sizeable sample of high-z QSOs can be used to explore their geometry, to isolate outflow dominated sources, and, eventually, to obtain more accurate estimates of their black hole masses.

\section{SUMMARY AND CONCLUSIONS}

We obtained spectropolarimetric observations of a sample of 25 QSOs extracted from the SDSS/DR7 catalog with $z<0.37$, the redshift limit at which the broad $\mathrm{H} \alpha$ line is visible in the SDSS spectra. We focused on the objects sufficiently bright to obtain good data quality with a single one hour observing block with FORS2 at the VLT: the median S/N of the total flux spectra in each spectral element is $\sim 300$. The objects were chosen to explore the QSO properties across the widest possible range of luminosities and BLR width (and consequently estimated black hole masses) by uniformly sampling the $L_{5100}-$ FWHM plane. We made separate selections of RL (9) and RQ (16) QSOs. As a result we obtained a representative sample of QSO with BLR widths spanning between 1,500 and $11,000 \mathrm{~km} \mathrm{~s}^{-1}$ and about two orders of magnitude in optical luminosity. There were no large changes in the spectra of these sources compared to the epoch in which SDSS observations were obtained, 10-15 years ago.

The median continuum polarization of sample is $0.59 \%$; the less luminous QSO $\left(\log \mathrm{L}_{5100}<44.5\right)$ are redder and less polarized $(\bar{P}=0.39 \%)$ than the more luminous ones $(\bar{P}=0.72 \%)$, most likely due to the dilution from the unpolarized starlight. Conversely, the presence of the narrow lines does not affect significantly the polarization properties. The comparison with previous observations of some sources of the sample indicate that the polarization is stable over a timescale of 30-40 years.

The integrated PA of the BLR is well aligned with the continuum PA, but the BLR is less polarized than the continuum: this is due to the PA swing across the BLR that produces significant cancellation. We also found that the BLR width in polarized light, $\mathrm{W}\left(\mathrm{I}_{P}\right)$, exceeds the line width in total flux, $\mathrm{W}(\mathrm{I})$, by a median factor $\sim 1.5$.

Several connections between the various polarization parame- 
ters emerge, e.g., between the PA swing across the BLR, the continuum polarization fraction and the increase of the line width in polarization with respect to its value in direct-light. All these results point to a simple explanation in which 1) the dominant polarization mechanism in QSO is due to scattering from material located in the equatorial plane, 2) the QSO polarization properties depend on their orientation. In particular a more face-on view results in low polarization, large PA swing across the BLR, and large polarized line broadening. Both the RQQs and RLQs exhibit similar relationship. RLQs show 1) a close alignment between their radio axis and both the continuum and BLR polarization and 2) a connection between radio core dominance and polarization, both indications of the role of orientation and equatorial scattering.

Nonetheless, there are three sources, all RL QSOs, in which the behavior is different: their polarized is redshifted by $\sim 2,000$ $\mathrm{km} \mathrm{s}^{-1}$ with respect to the total flux BLR, but with a BLR width essentially unchanged.

The information derived from spectropolarimetry can be used to mitigate the inclination bias in the virial estimates of the black hole masses. One can use the $\mathrm{W}\left(\mathrm{I}_{P}\right) / \mathrm{W}(\mathrm{I})$ ratio as correction factor for the measured FWHM, as this directly measures the increase in the velocity spread from the vantage (equatorial) point of view of the scatterers. In the sample of QSOs studied here the correction factor can be as large as $\sim 6$. The dependence of $\mathrm{W}\left(\mathrm{I}_{P}\right) / \mathrm{W}(\mathrm{I})$ on the continuum polarization indicates that the correction is stronger for the least polarized sources. Significant care is needed to obtain a robust measurement of this broadening effect: we adopted as measurement of the line width the separation of the $25^{\text {th }}$ and $75^{\text {th }}$ percentiles of the line profile, a definition that is robust against the uncertainties due to the low $\mathrm{S} / \mathrm{N}$ and of the positive bias of the polarization data.

Fig. 17 shows a short summary, in the form of a flow chart, which relates the various observed polarization properties, and their physical implications, which lead to the implied polarization scenario. It appears that the polarization of both the continuum and the BLR are produced by an equatorial scattering medium which resides close to, or possibly at, the BLR. This scattering geometry explains the polarization properties of this representative sample of QSO. The polarization properties can be used to explore the nature and the geometry of the scattering medium in type 1 AGN, and eventually understand its origin. In addition, spectropolarimetry may provide a useful tool to improve the accuracy of the black hole mass estimates.

\section{ACKNOWLEDGMENTS}

This research was supported by the Israel Science Foundation (grant no. 1008/18).

\section{DATA AVAILABILITY}

The data underlying this article will be shared on reasonable request to the corresponding author.

\section{APPENDIX A: THE INTERSTELLAR POLARIZATION}

Thermal emission from the dust grains is polarized (Hildebrand et al. 1999) and Planck Collaboration XXI (2015) have shown that the polarization at $353 \mathrm{GHz}$ measured by the Planck satellite is closely connected with the optical polarization

\section{The Polarisation implications flow chart}

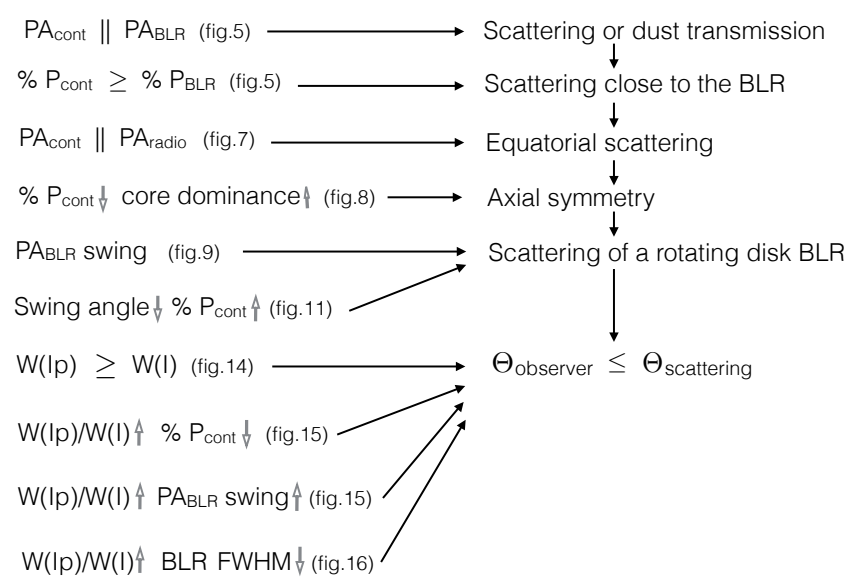

Figure 17. Summary of the the observed polarization properties, which all lead to a consistent interpretation. The polarization of unobscured quasars is produced by scattering, the scattering medium is axially symmetric, equatorial, and resides at the outskirts of the BLR.

of stars. More specifically they found that 1) the sub-millimeter polarization is perpendicular to that measured in the optical and 2) the ratio $R_{S / V}=p_{S} /\left(p_{V} / \tau_{V}\right)$ (where $p_{s}$ and $p_{V}$ are the degree of polarization in the sub-mm and $\mathrm{V}$ band, respectively, and $\tau_{V}$ is the absorption optical depth) is fairly constant across different lines of sight in the diffuse interstellar medium with a measured value of $R_{S / V}=4.2 \pm 0.2$ (stat.) \pm 0.3 (syst.).

We estimated the contamination level of the quasar polarization from the ISP following the analysis of Pelgrims (2019). At the location of each of the 25 QSOs we measured the Stokes parameters of the $353 \mathrm{GHz}$ emission after smoothing the images with a Gaussian with a FWHM of $15^{\prime}$. The expected contribution to the optical polarization is estimated as $q_{V}=-\left(q_{S} \tau_{V}\right) / R_{S / V}$ and $u_{V}=-\left(u_{S} \tau_{V}\right) / R_{S / V}$ and it can be subtracted in the Stokes parameters space from the observed QSO polarization.

The actual effect on our data must be estimated by considering the wavelength dependence of the ISP to transform the ISP polarization in the $\mathrm{V}$ band to the wavelength of the $\mathrm{H} \alpha$ line in each individual QSO. We used the empirical law from Serkowski et al. (1975) adopting as wavelength at which the ISP reaches its maximum $\lambda_{\max }=5500 \AA$. This correction is fairly small, with a ratio between $p_{\mathrm{H} \alpha}$ and $p_{V}$ ranging from 0.76 to 0.94 .

In Fig. A1 (top panel) we compare the observed continuum polarization with the estimated value of the ISP. The other two panels show instead the effects of the subtraction of the ISP on $P_{\text {cont. }}$ and $\mathrm{PA}_{\mathrm{cont}}$. Overall, these measurements are consistent within the errors. The only exceptions are J154019, J155444, and J214054 where the observed and corrected percentage of polarization differ by more than $2 \sigma$. For these objects we applied the ISP correction.

We report in the Table below the observed and corrected value for these sources.

\section{REFERENCES}

Afanasiev V. L., Popović L. Č., Shapovalova A. I., 2019, MNRAS, 482, 4985

Agol E., Blaes O., 1996, MNRAS, 282, 965 
Table A1. ISP corrections

\begin{tabular}{lrrrr}
\hline Name & $P$ cont. (\%) & PA cont. & $P$ corr. (\%) & PA corr. \\
\hline $\mathrm{J} 154019$ & $1.13 \pm 0.11$ & $79 \pm 3$ & $2.03 \pm 0.26$ & $89 \pm 4$ \\
$\mathrm{~J} 155444$ & $1.50 \pm 0.06$ & $84 \pm 1$ & $1.23 \pm 0.09$ & $86 \pm 2$ \\
$\mathrm{~J} 214054$ & $0.40 \pm 0.07$ & $133 \pm 5$ & $0.67 \pm 0.12$ & $131 \pm 5$ \\
\hline
\end{tabular}

Column description: 1) source name, 2) and 3) observed percentage of polarization and polarization position angle of the continuum at $6563 \AA$, 4) and 5) percentage of polarization and polarization position angle after ISP subtraction.

Alexandroff R. M., Zakamska N. L., Barth A. J., Hamann F., Strauss M. A., Krolik J., Greene J. E., Pâris I., Ross N. P., 2018, MNRAS, 479, 4936

Antonucci R., 1993, ARA\&A, 31, 473

Antonucci R. R. J., 1983, Nature, 303, 158

Antonucci R. R. J., Miller J. S., 1985, ApJ, 297, 621

Baldi R. D., Capetti A., Robinson A., Laor A., Behar E., 2016, MNRAS, 458, L69

Baskin A., Laor A., 2005, MNRAS, 356, 1029

Baskin A., Laor A., 2018, MNRAS, 474, 1970

Becker R. H., White R. L., Helfand D. J., 1995, ApJ, 450, 559

Berriman G., Schmidt G. D., West S. C., Stockman H. S., 1990, ApJS, 74, 869

Boroson T. A., Green R. F., 1992, ApJS, 80, 109

Calderone G., Foschini L., Ghisellini G., Colpi M., Maraschi L., Tavecchio F., Decarli R., Tagliaferri G., 2011, MNRAS, 413, 2365

Chang S.-J., Heo J.-E., Di Mille F., Angeloni R., Palma T., Lee H.-W., 2015, ApJ, 814, 98

Cohen M. H., Ogle P. M., Tran H. D., Vermeulen R. C., Miller J. S., Goodrich R. W., Martel A. R., 1995, ApJ, 448, L77

Collin S., Kawaguchi T., Peterson B. M., Vestergaard M., 2006, A\&A, 456, 75

Condon J. J., Cotton W. D., Greisen E. W., Yin Q. F., Perley R. A., Taylor G. B., Broderick J. J., 1998, AJ, 115, 1693

Corbett E. A., Robinson A., Axon D. J., Young S., 2000, MNRAS, 319,685

de Vries W. H., Barthel P. D., O’Dea C. P., 1997, A\&A, 321, 105

Fine S., Croom S. M., Hopkins P. F., Hernquist L., BlandHawthorn J., Colless M., Hall P. B., Miller L., Myers A. D., Nichol R., Pimbblet K. A., Ross N. P., Schneider D. P., Shanks T., Sharp R. G., 2008, MNRAS, 390, 1413

Fine S., Jarvis M. J., Mauch T., 2011, MNRAS, 412, 213

Fischer T. C., Crenshaw D. M., Kraemer S. B., Schmitt H. R., 2013, ApJS, 209, 1

Gaskell C. M., Goosmann R. W., Merkulova N. I., Shakhovskoy N. M., Shoji M., 2012, ApJ, 749, 148

Goodrich R. W., Miller J. S., 1988, ApJ, 331, 332

Goodrich R. W., Miller J. S., 1989, ApJ, 346, L21

Goodrich R. W., Miller J. S., 1994, ApJ, 434, 82

Helfand D. J., White R. L., Becker R. H., 2015, ApJ, 801, 26

Hildebrand R. H., Dotson J. L., Dowell C. D., Schleuning D. A., Vaillancourt J. E., 1999, ApJ, 516, 834

Hook R., Romaniello M., Péron M., Ballester P., Gabasch A., Izzo C., Ullgrén M., Maisala S., Oittinen T., Solin O., Savolainen V., Järveläinen P., Tyynelä J., 2008, in Argyle R. W., Bunclark P. S., Lewis J. R., eds, Astronomical Data Analysis Software and Systems XVII Vol. 394 of Astronomical Society of the Pacific Conference Series, ESO Reflex: A Graphical Workflow Engine for Data Reduction. p. 638
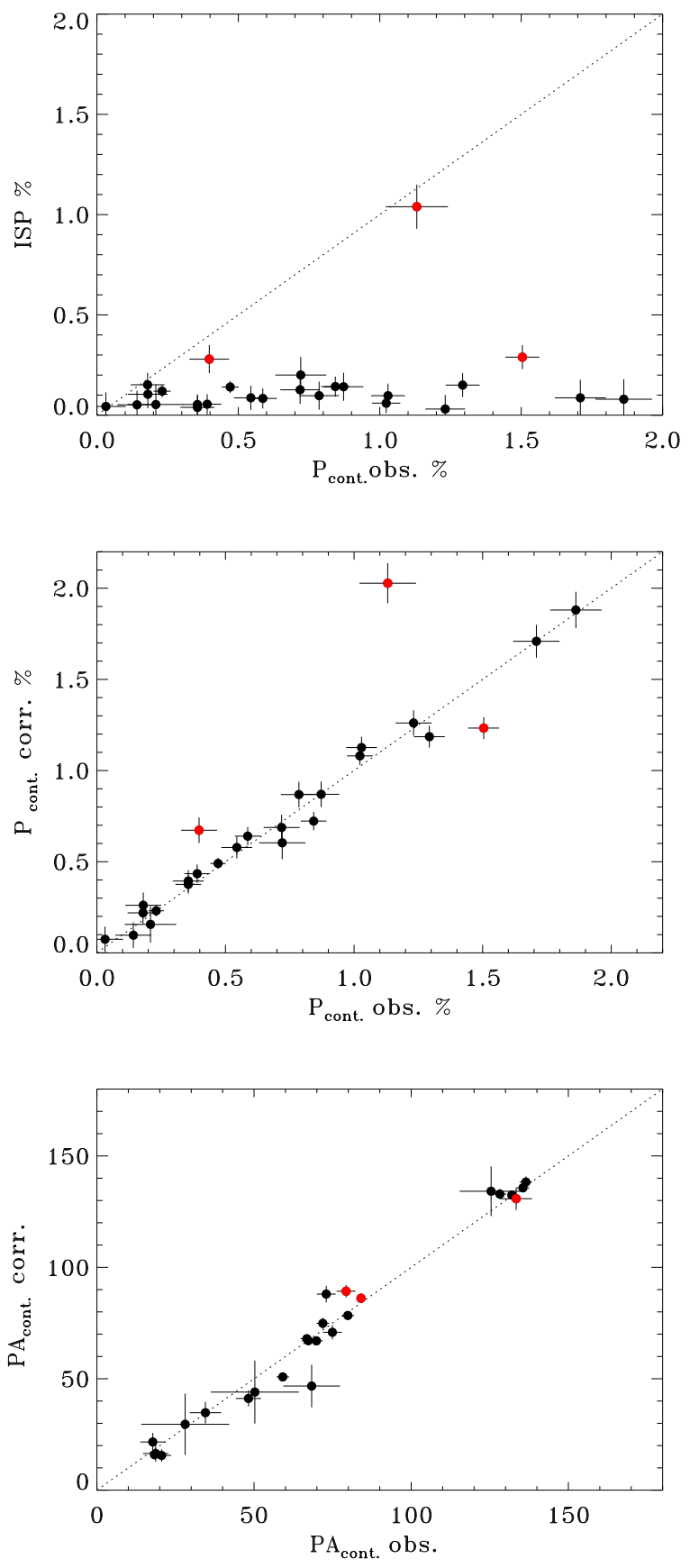

Figure A1. Top: comparison between the observed continuum polarization and the expected contribution from the ISP. Comparison of the observed continuum polarization (middle) and PA (bottom) with the values after correction for the ISP contribution. We mark in red the three sources (namely J154019, J155444, and J214054) where the observed and corrected percentage of polarization differ by more than $2 \sigma$. For these objects we applied the ISP correction.

Jiang L., Fan X., Ivezić Ž., Richards G. T., Schneider D. P., Strauss M. A., Kelly B. C., 2007, ApJ, 656, 680

King A., Pounds K., 2015, ARA\&A, 53, 115

Kishimoto M., Antonucci R., Boisson C., Blaes O., 2004, MN-

RAS, 354, 1065

Korista K., Ferland G., 1998, ApJ, 495, 672 
Laor A., Netzer H., Piran T., 1990, MNRAS, 242, 560

Lee H. W., Blandford R. D., 1997, MNRAS, 288, 19

Lee H.-W., Yun J.-H., 1998, MNRAS, 301, 193

Lira P., Goosmann R. W., Kishimoto M., Cartier R., 2020, MN-

RAS, 491, 1

Marin F., 2014, MNRAS, 441, 551

Mathewson D. S., Ford V. L., 1970, MmRAS, 74, 139

Miller J. S., Antonucci R. R. J., 1983, ApJ, 271, L7

Ogle P. M., Cohen M. H., Miller J. S., Tran H. D., Goodrich R. W., Martel A. R., 1999, ApJS, 125, 1

Oshlack A. Y. K. N., Webster R. L., Whiting M. T., 2001, ApJ, 558, 578

Patat F., Romaniello M., 2006, PASP, 118, 146

Pelgrims V., 2019, A\&A, 622, A145

Planck Collaboration XXI 2015, A\&A, 576, A106

Robinson A., Corbett E. A., Axon D. J., Young S., 1999, MNRAS, 305, 97

Rojas Lobos P. A., Goosmann R. W., Hameury J. M., Marin F., 2020, A\&A, 637, A88

Savić D., Goosmann R., Popović L. Č., Marin F., Afanasiev V. L., 2018, A\&A, 614, A120

Schmidt G. D., Hines D. C., 1999, ApJ, 512, 125

Schneider D. P., Richards G. T., Hall P. B., Strauss M. A., Anderson S. F., Boroson T. A., Ross N. P., Shen Y., Brandt W. N., Fan X., Inada N., Jester S., Knapp G. R., Krawczyk C. M., Thakar A. R., Vanden Berk D. E., 2010, AJ, 139, 2360

Serkowski K., Mathewson D. L., Ford V. L., 1975, ApJ, 196, 261

Shen Y., Richards G. T., Strauss M. A., Hall P. B., Schneider D. P., Snedden S., Bizyaev D., Brewington H., Malanushenko V., Malanushenko E., Oravetz D., Pan K., Simmons A., 2011, ApJS, 194, 45

Smith J. E., Robinson A., Alexander D. M., Young S., Axon D. J., Corbett E. A., 2004, MNRAS, 350, 140

Smith J. E., Robinson A., Young S., Axon D. J., Corbett E. A., 2005, MNRAS, 359, 846

Smith J. E., Young S., Robinson A., Corbett E. A., Giannuzzo M. E., Axon D. J., Hough J. H., 2002, MNRAS, 335, 773

Stern J., Laor A., 2012, MNRAS, 423, 600

Stockman H. S., Angel J. R. P., Miley G. K., 1979, ApJ, 227, L55

Stockman H. S., Moore R. L., Angel J. R. P., 1984, ApJ, 279, 485

Vaillancourt J. E., 2006, PASP, 118, 1340

Vernet J., Fosbury R. A. E., Villar-Martín M., Cohen M. H., Cimatti A., di Serego Alighieri S., Goodrich R. W., 2001, A\&A, 366,7

Wardle J. F. C., Kronberg P. P., 1974, ApJ, 194, 249

Whittle M., 1985, MNRAS, 213, 33

Wills B. J., Browne I. W. A., 1986, ApJ, 302, 56

Young S., Axon D. J., Robinson A., Hough J. H., Smith J. E., 2007, Nature, 450, 74

Zubko V. G., Laor A., 2000, ApJS, 128, 245 
APPENDIX B: SUPPLEMENTARY MATERIAL 

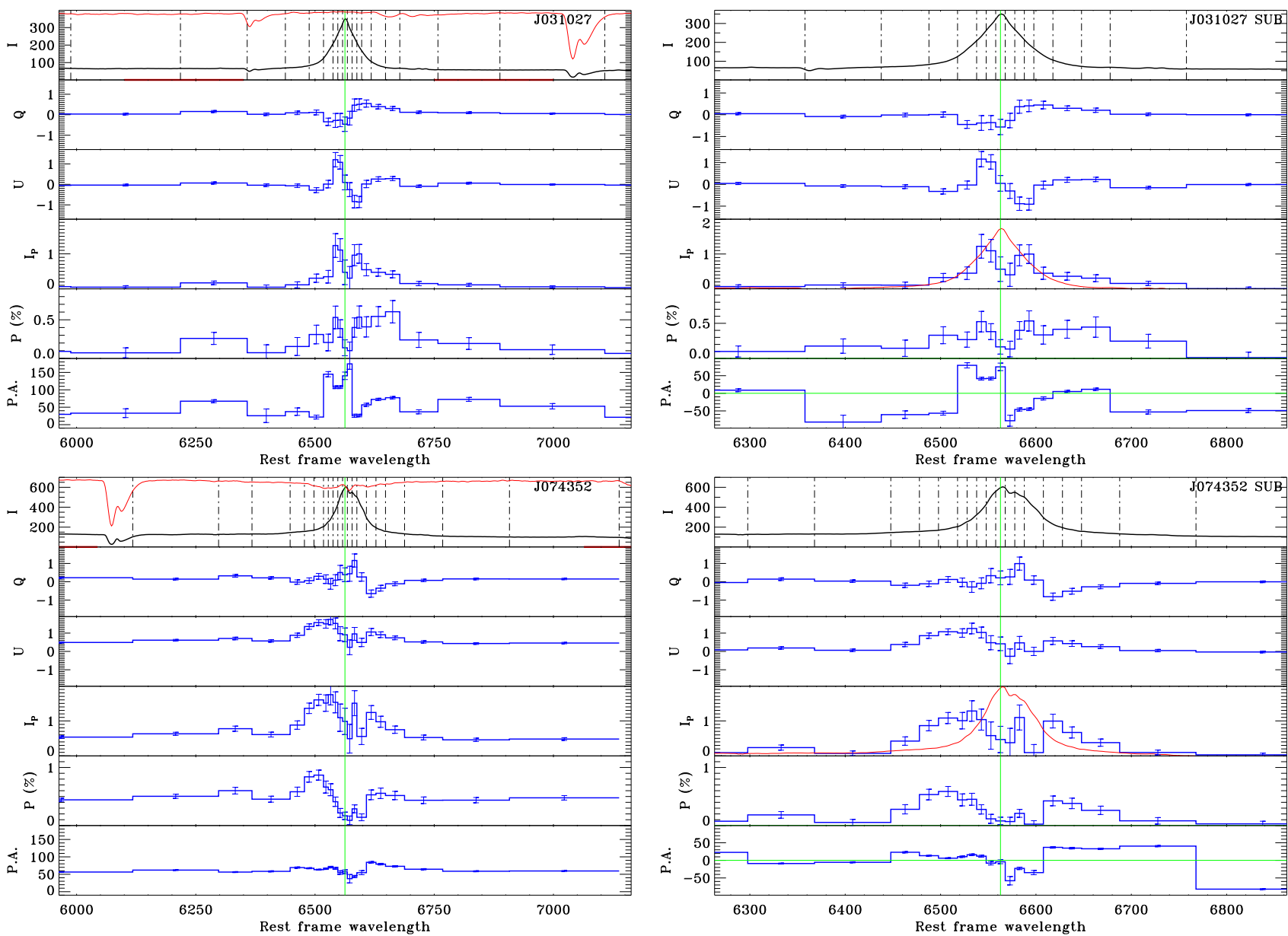

Left: top three panels: I, Q, and U Stokes parameters shown over a spectral region of $1200 \AA$. I is shown at full spectral resolution, the vertical dashed lines mark the boundaries of the regions used for the rebinning. Wavelengths are rest frame in Å, fluxes are in arbitrary units. The green vertical line marks the location of the $\mathrm{H} \alpha$ line. The red curve in the top panel represents the ratio between the SDSS and the corrected VLT spectra in which can be located the telluric absorption bands. The two horizontal red lines locate the regions used to estimate the continuum polarization. Bottom three panels: polarized flux $I_{P}$ , percentage of polarization $P$, and polarization position angle P.A.. Right: same as the left panels, but after subtraction of the continuum polarization and with a reduced spectral region $(600 \AA)$. On the polarized flux we overplotted, in red, the total intensity spectrum. In the $P$ panel, the horizontal line shows the continuum $P$ at the BLR center. Angles are measured with respect to the continuum P.A.. 

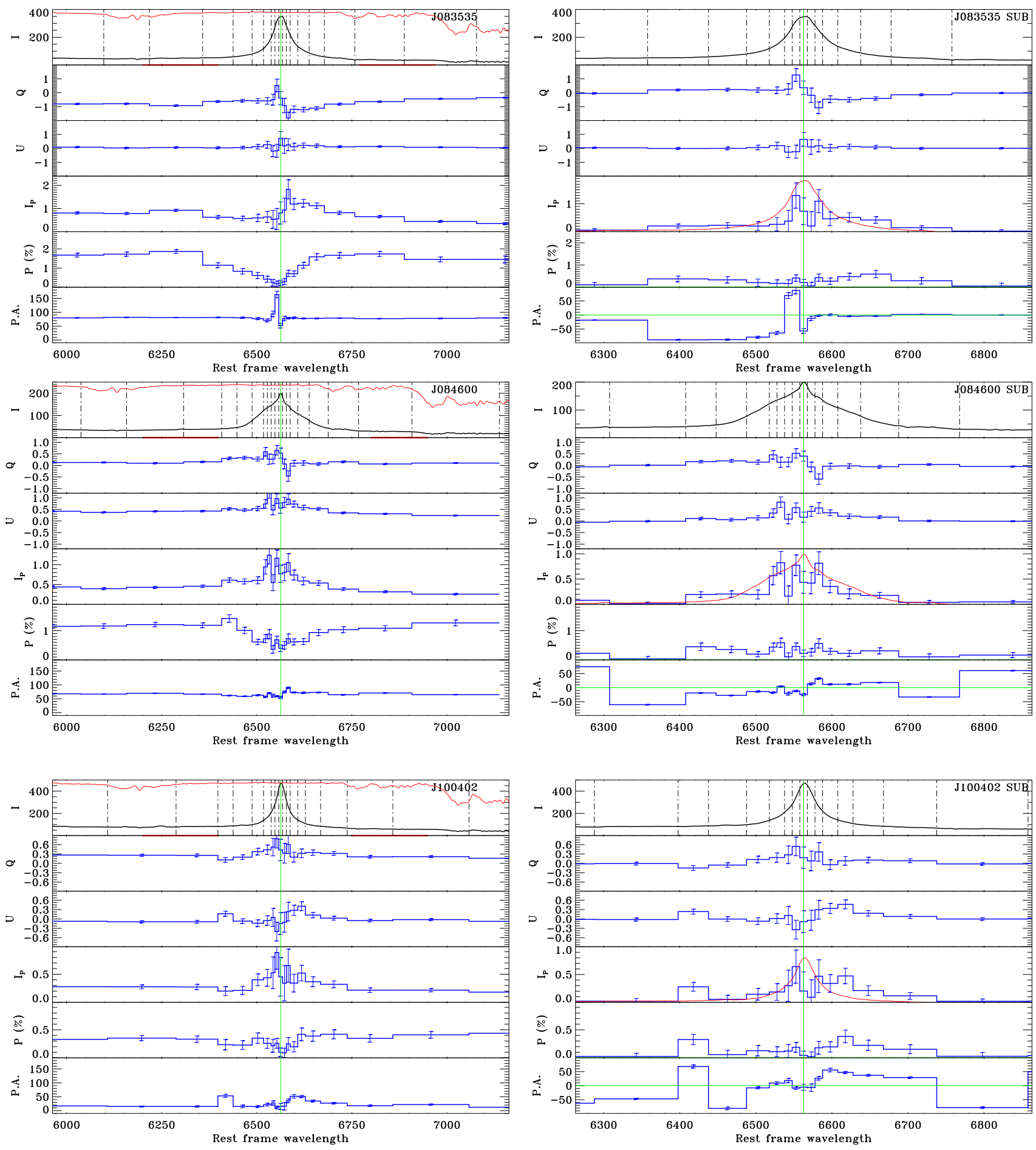

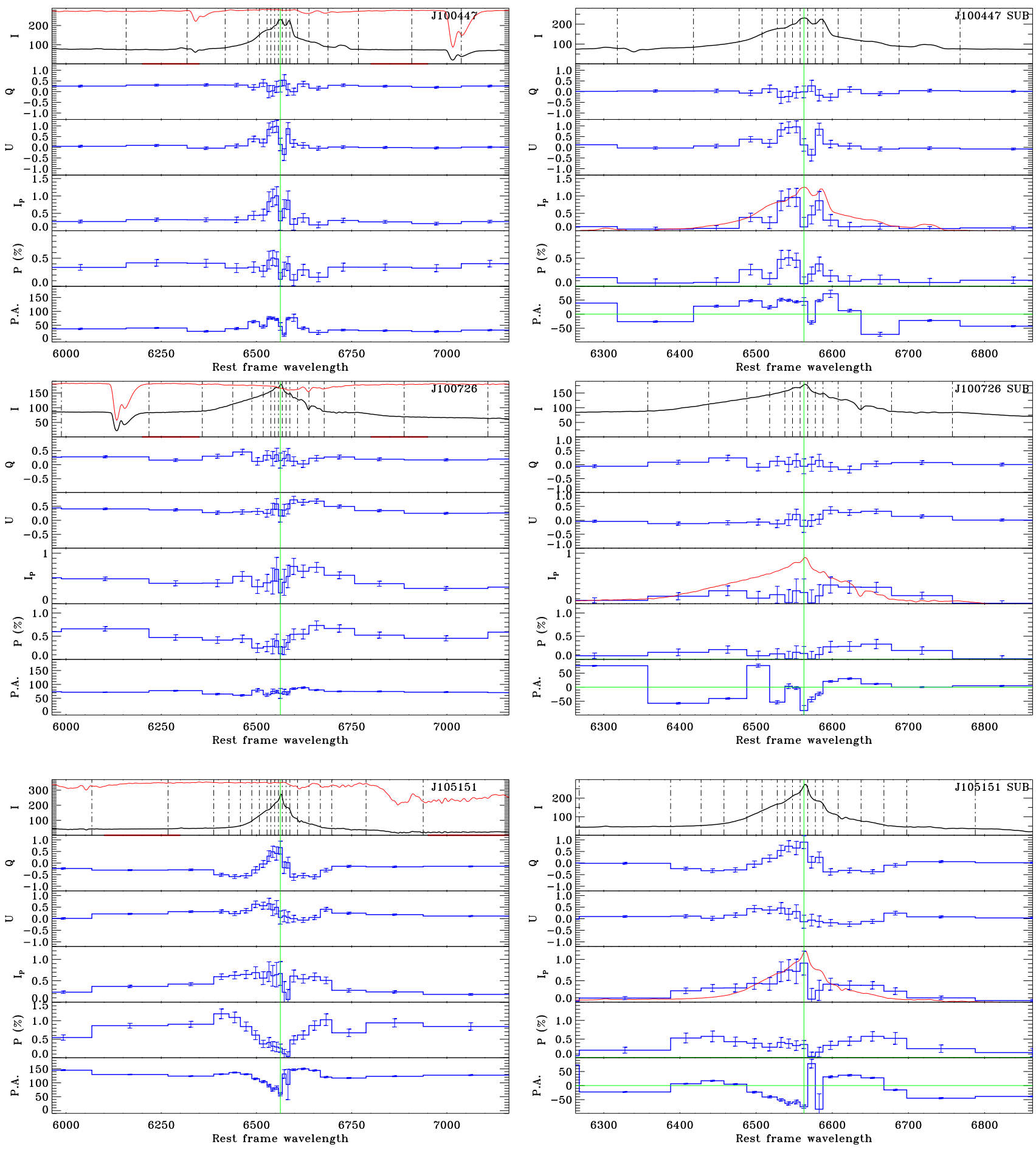

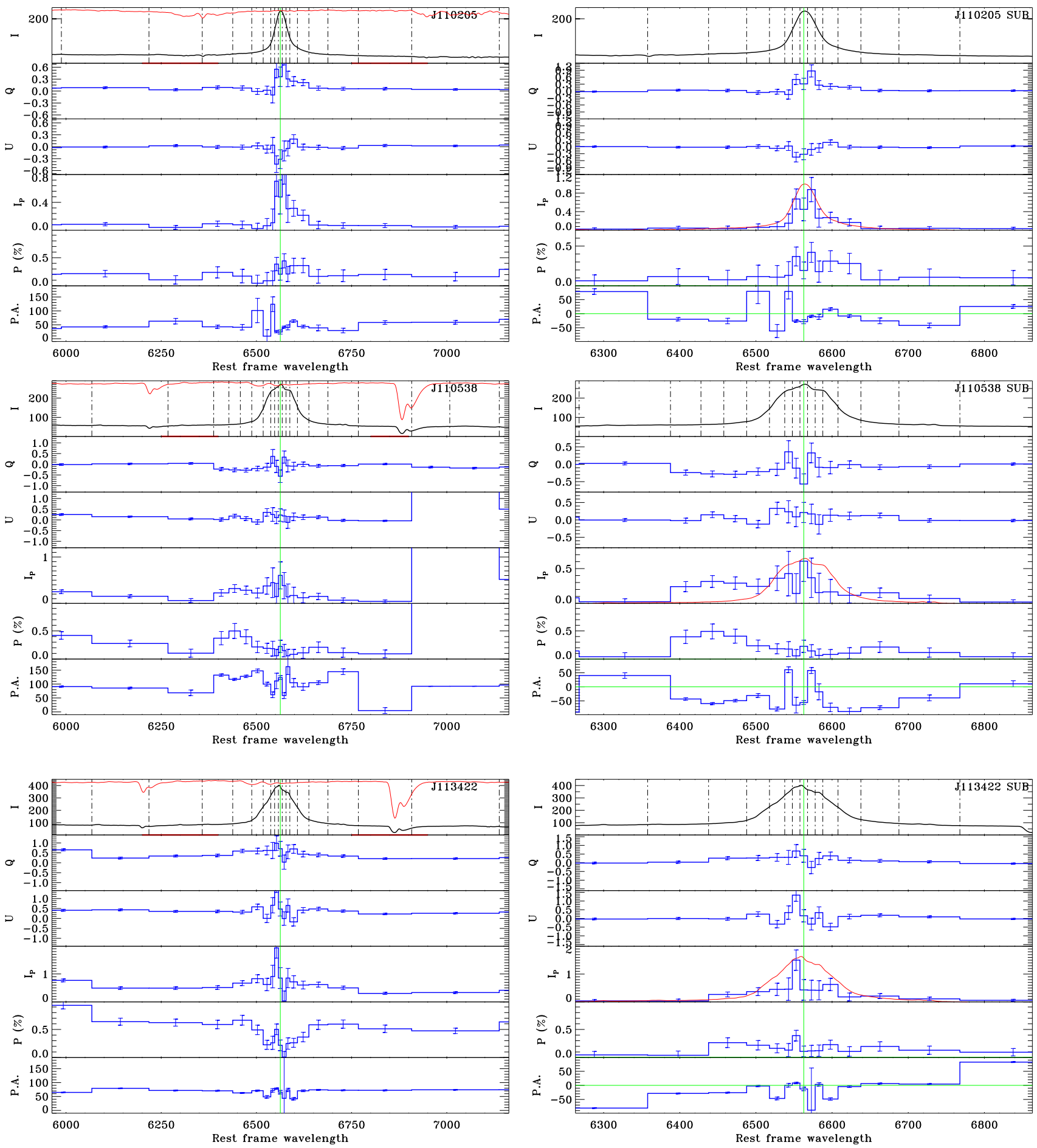

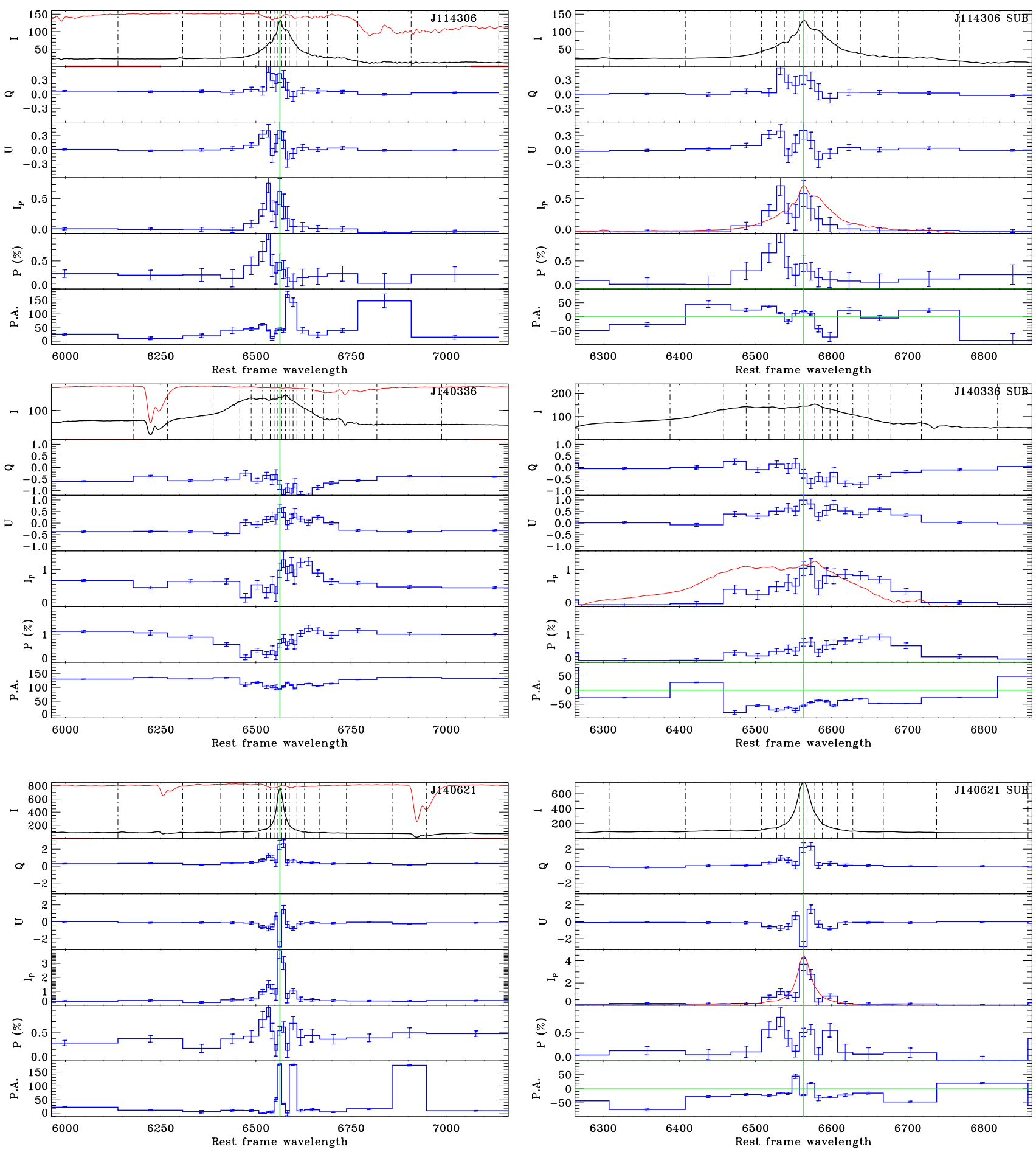

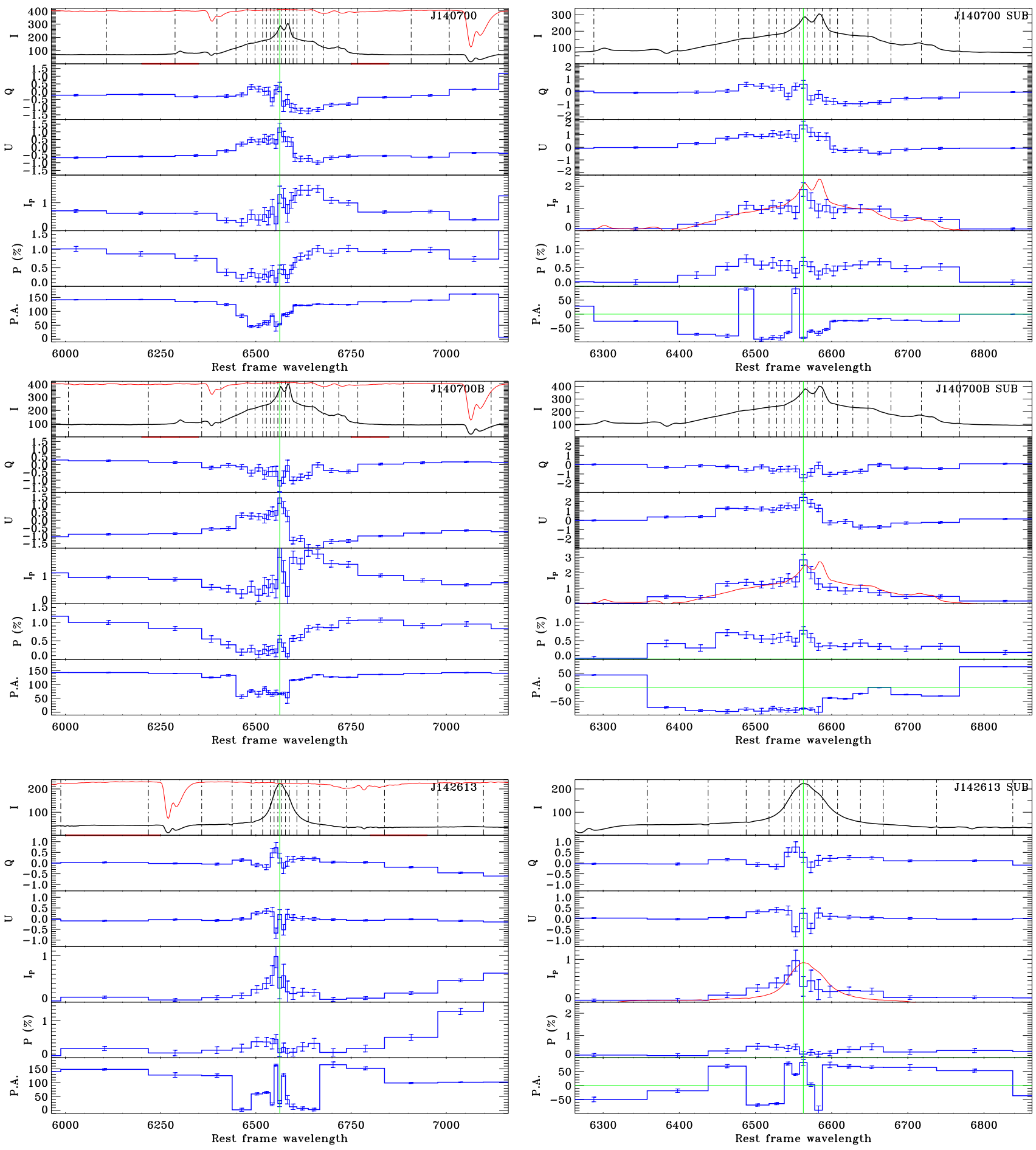

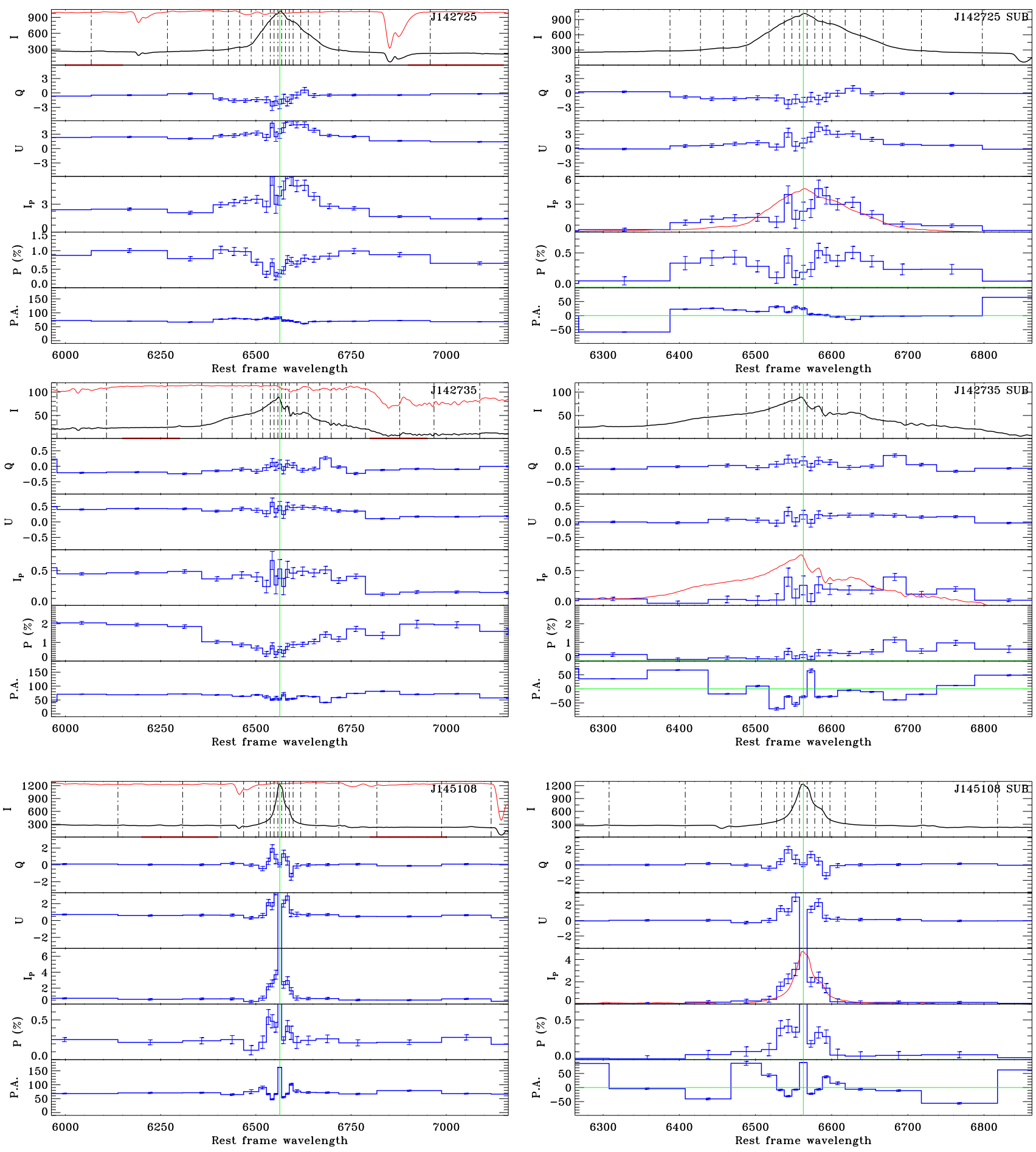

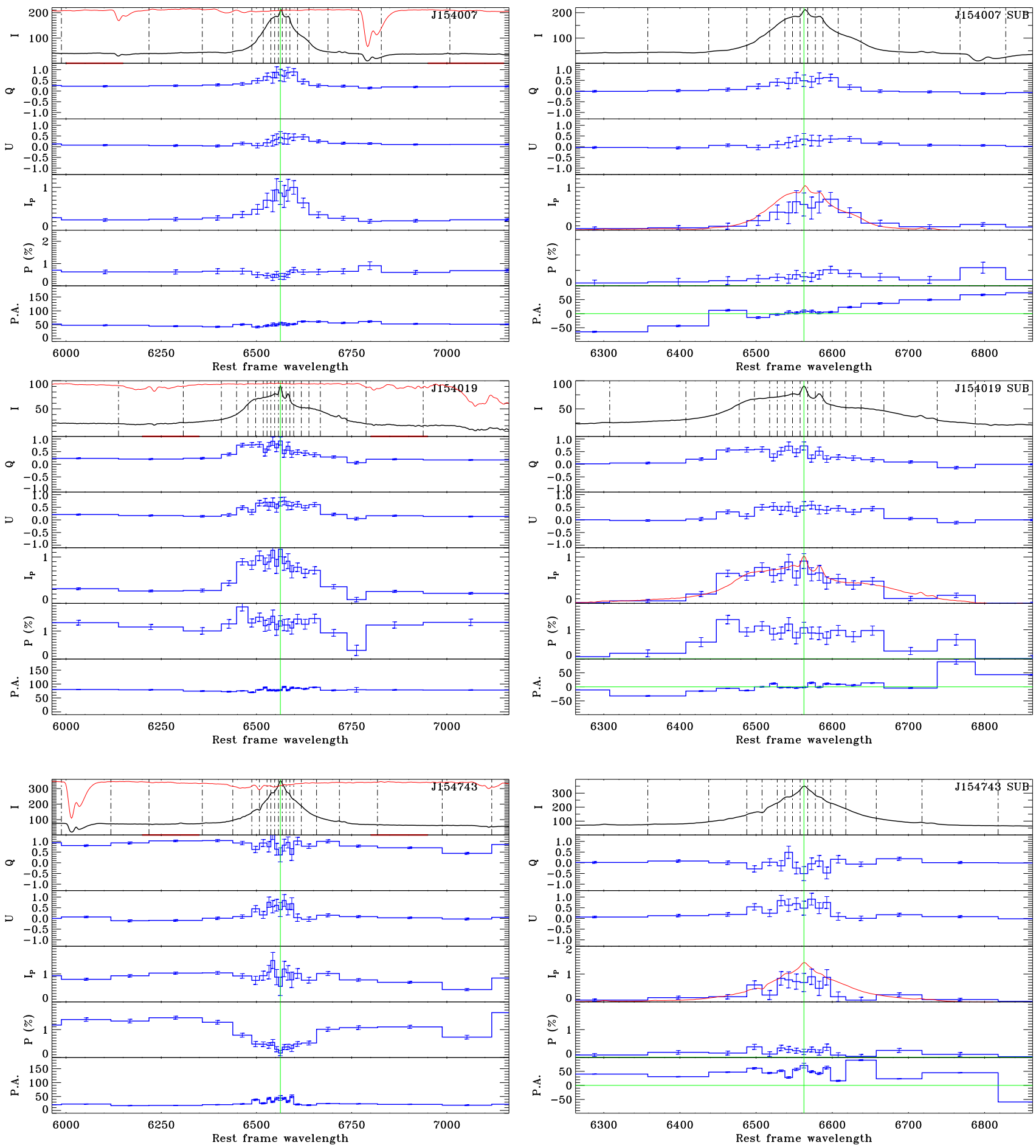

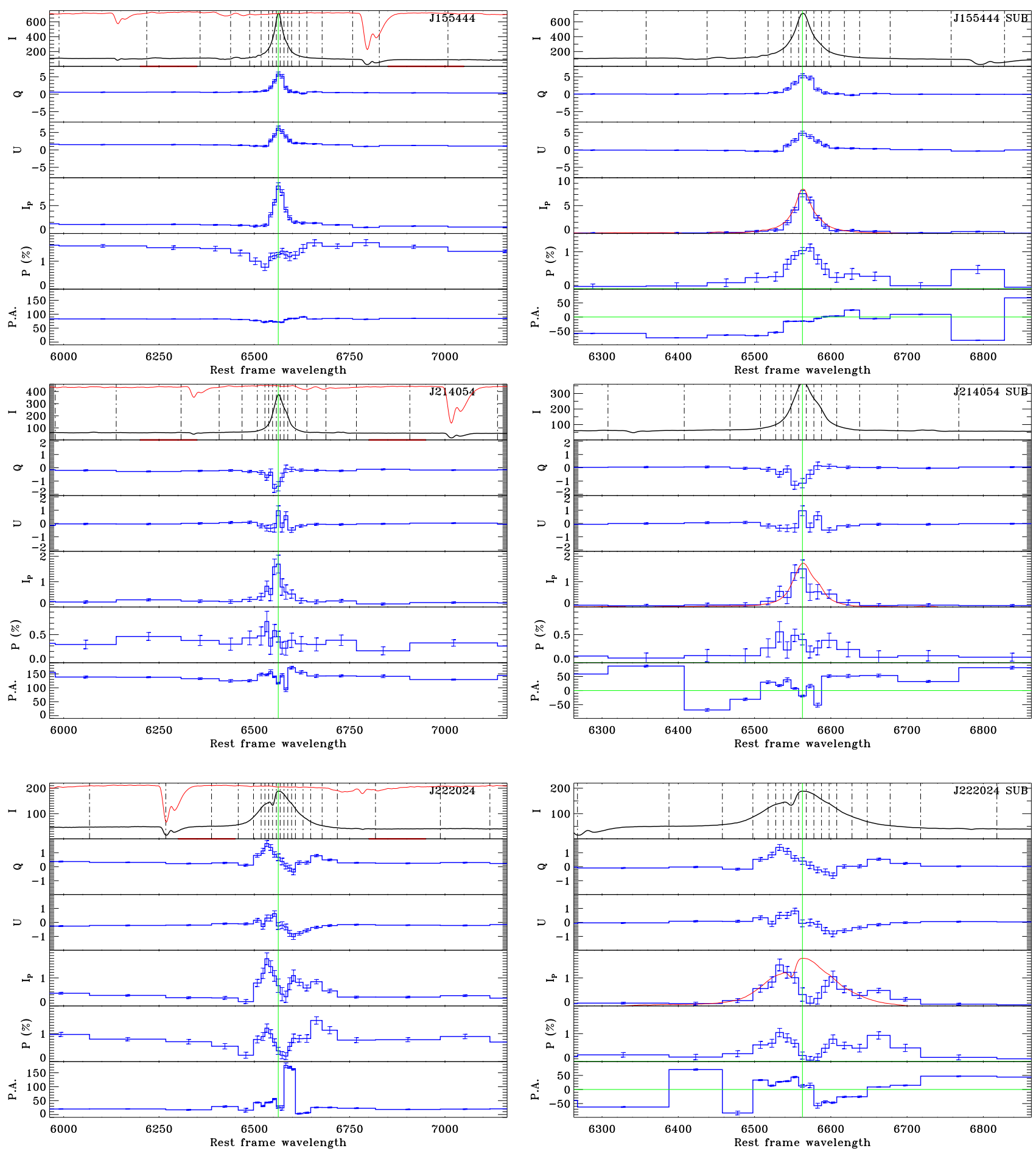


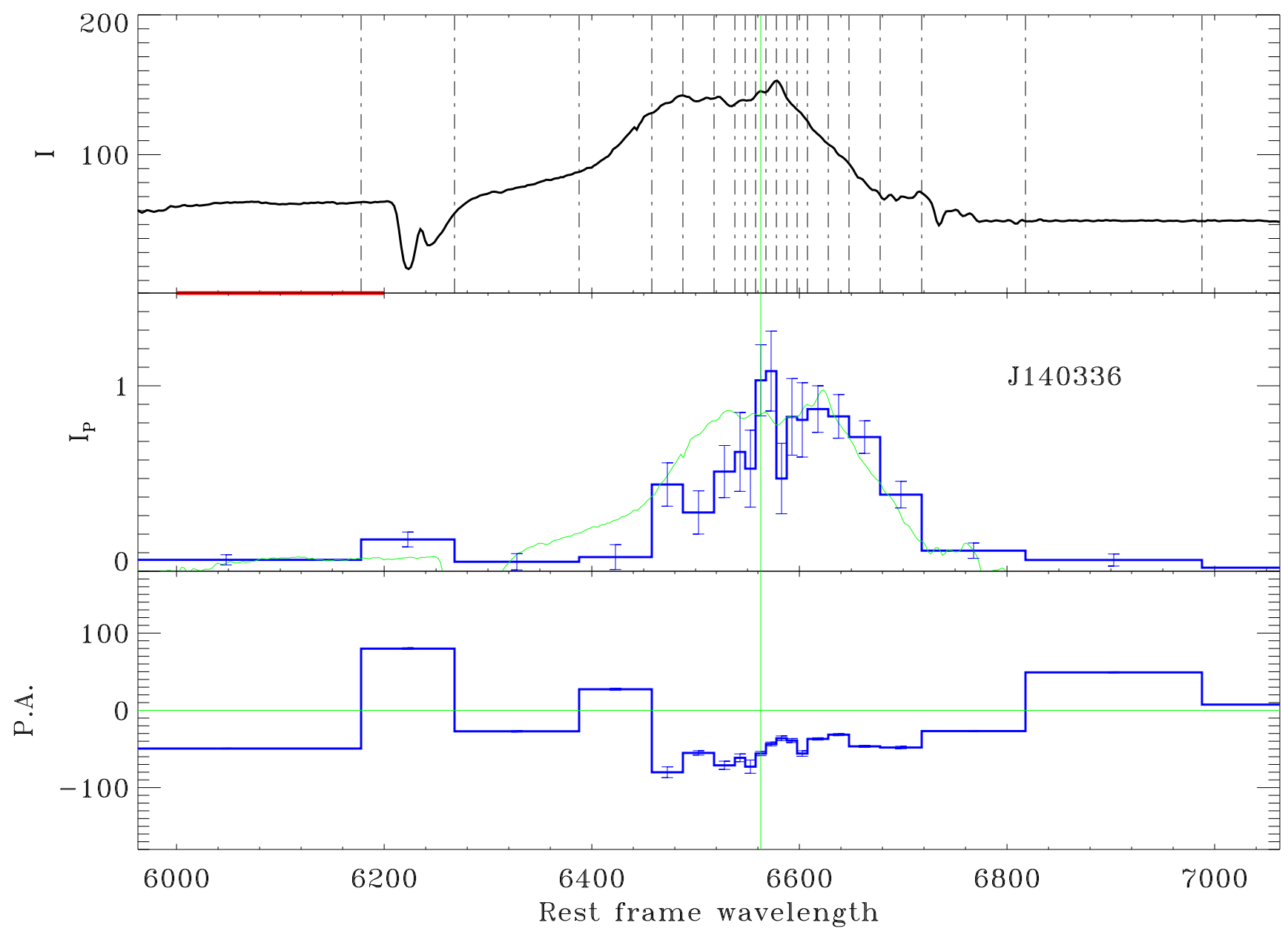

